\title{
En-gauging naturalness
}

\author{
Aoife Bharucha $^{1,2, a}$, Andreas Goudelis ${ }^{3, b}$, Moritz McGarrie $^{4, c}$ \\ ${ }^{1}$ II. Institut für Theoretische Physik, Universität Hamburg, Luruper Chaussee 149, 22761 Hamburg, Germany \\ 2 Physik Department T31, Technische Universität München, James-Franck-Straße 1, 85748 Garching, Germany \\ ${ }^{3}$ LAPTh, Université de Savoie, CNRS, 9 Chemin de Bellevue, B.P. 110, 74941 Annecy-le-Vieux, France \\ ${ }^{4}$ Deutsches Elektronen-Synchrotron, DESY, Notkestrasse 85, 22607 Hamburg, Germany
}

Received: 13 February 2014 / Accepted: 9 April 2014 / Published online: 20 May 2014

(C) The Author(s) 2014. This article is published with open access at Springerlink.com

\begin{abstract}
The discovery of a $125.5 \mathrm{GeV}$ Higgs with standard model-like couplings and naturalness considerations motivate gauge extensions of the MSSM. We analyse two variants of such an extension and carry out a phenomenological study of regions of the parameter space satisfying current direct and indirect constraints, employing state-of-theart two-loop RGE evolution and GMSB boundary conditions. We find that due to the appearance of non-decoupled D-terms it is possible to obtain a $125.5 \mathrm{GeV}$ Higgs with stops below $2 \mathrm{TeV}$, while the uncoloured sparticles could still lie within reach of the LHC. We compare the contributions of the stop sector and the non-decoupled D-terms to the Higgs mass, and study their effect on the Higgs couplings. We further investigate the nature of the next-to lightest supersymmetric particle, in light of the GMSB motivated searches currently being pursued by ATLAS and CMS.
\end{abstract}

\section{Introduction}

The recent discovery of a $\sim 125.5 \mathrm{GeV}$ particle consistent with the properties of the standard model Higgs boson [1,2] and no direct evidence of supersymmetry (SUSY) in the current LHC data are pushing traditional setups of gaugemediated supersymmetry breaking (GMSB) for the minimal supersymmetric standard model (MSSM) into fine-tuned territory. In the MSSM, to obtain a lightest CP-even scalar of the observed mass requires either heavy stops, thereby introducing a naturalness or fine-tuning problem, or substantial left-right stop mixing, which, being strongly dependent on the trilinear soft term $A_{\mathrm{t}}$, is heavily influenced by the mechanism of supersymmetry-breaking mediation that is invoked. In particular, in GMSB trilinear terms such as $A_{\mathrm{t}}$ are vanish-

\footnotetext{
a e-mail: aoife.bharucha@tum.de

b e-mail: andreas.goudelis@1apth.cnrs.fr

c e-mail: moritz.mcgarrie@desy.de
}

ing at the supersymmetry-breaking scale $M$, and a large $A_{\mathrm{t}}$ can only be generated via renormalisation group evolution. This requires the scale $M$ to be very high, which is also detrimental to the naturalness of the theory. Moreover, in minimal SUSY models, choosing heavy stops results in the sparticle spectrum becoming heavier and beyond the reach of the LHC, and consequently phenomenologically less interesting.

The heart of the problem appears to be that in the MSSM, the tree-level Higgs mass is simply too small, the upper bound being the mass of the $Z$ boson. If, however, we drop minimality from our criteria, natural scenarios of supersymmetry breaking with discovery potential still persist. These usually involve lifting the Higgs potential at tree-level through nondecoupled F- or D-type terms [3-5], the later of which arise when the MSSM is extended by additional gauge groups. The less-studied gauge extensions involving non-decoupled D-terms could enhance the tree-level Higgs mass, resulting in detectable deviations in the Higgs couplings, and further induce suppressions to the scalar masses compared to minimal GMSB. Such models may therefore have a direct impact on phenomenology and, unsurprisingly, have as of lately found increasing interest [6-16].

In this paper we study a quiver model or gauge extension of the MSSM within the framework of GMSB, to determine whether it is indeed possible to reproduce the observed Higgs boson mass while keeping the stop masses below roughly $2 \mathrm{TeV}$. In particular we build a tailor-made spectrum generator for our model using the publicly available tool SARAH [17-19]. This allows us to perform the renormalisation group evolution at two loops and analyse several aspects of the model's phenomenology. Although we do not carry out a thorough "naturalness" or fine-tuning study, it is at least clear that qualitatively, having stops lighter than benchmark minimal GMSB certainly improves the relative naturalness of the model. We therefore study the resulting spectra for the model, consistent with experimental results, in particular the Higgs sector. We further investigate possible signatures of 
this model at the LHC, taking into account the latest results of GMSB motivated searches.

It is useful to summarise the current status of the literature that explores two-site or minimal quiver models. Initially a simple two-site deconstruction, similar to our model MI (described later in Sect. 3.1), with gauge-mediated boundary conditions was proposed in [7], in particular in which both quiver gauge groups $G_{A}$ and $G_{B}$ are $\mathrm{U}(1) \times \mathrm{SU}(2) \times \mathrm{SU}(3)$ and in which both site $\mathrm{A}$ and $\mathrm{B}$ gauge couplings unify separately. As in such models sfermion soft masses are typically smaller than gaugino soft masses it unwittingly allows for the foundations of a natural spectrum. However the resulting tree-level Higgs mass is described by the MSSM, such that the observed Higgs mass [1,2] must therefore be generated by large $A_{\mathrm{t}}$ or heavy stops. Later in [10] the non-decoupled D-terms of [3] in the Higgs sector were included by hand, although they did not contribute significantly and the resulting Higgs mass of their benchmarks, of around $116 \mathrm{GeV}$, are also now firmly excluded. In [8,9] a model similar to our model MII ${ }^{1}$ was sketched in which it is argued that one could obtain (a) a linking field VEV $v<10 \mathrm{TeV}$ (b) unification of each gauge site separately, as well as (c) lighter third generation scalars relative to first and second generation ones, due to location of generations in different sites. Most of the studies so far mentioned were carried out at tree-level. The formulae for the soft masses in the case of gauge mediation were also given in [12,23,24] where some interest had developed in finding and making precise models where sfermions were lighter than gauginos at the messenger scale. In light of the Higgs discovery [1,2] and naturalness, the split families models of [8,9] have re-emerged [13,14,25].

In [14] a study using the MSSM spectrum generator, SOFTSUSY [26] combined with some private codes found that the model based on [3,8-10,12,13,23-25] could not simultaneously achieve unification and obtain the necessary enhancement of the Higgs mass. If the necessary enhancement was obtained then the gauge groups hit a Landau pole much before the GUT scale. Some models involving three sites were proposed to alleviate the problem. The study of [14] misses some leading order effects to some one-loop RGEs and as such a more comprehensive study and implementation of these models is necessary. Furthermore, simpler (more minimal) variants of this class of model may fair better. The core issue is that the $\mathrm{SU}(3)_{A} \times \mathrm{SU}(3)_{B}$ matter component of the quiver introduces additional flavours to each side, respectively, charged also under the electroweak groups, and this additional matter content can cause the Landau pole problem, if one wishes for $g_{A}>g_{B}$ to increase the effect of the Higgs enhancement. Given the importance of the Higgs enhancement, perhaps it is worth removing the

\footnotetext{
1 The first references of split families we are aware of are in [20-22], in a non-SUSY context.
}

copy of SU(3) (and possibly complete unification) in favour of naturalness. Such a setup should anyway test what is the maximum allowed enhancement to the Higgs from the electroweak quiver without worrying about unification or Landau poles.

There are clearly a number of important and unresolved issues which our paper addresses. The most detailed study so far, in [14], although useful does not include some leading one-loop contributions to the RGEs (see Appendix A and [27]), it is essentially based on an MSSM spectrum generator [26] such that the Higgs enhancement is added afterwards by hand. Considering the importance not only of Higgs observables but the pressing issue of naturalness this is not sufficient, and a dedicated (and publicly available) spectrum generator and study of this type of model are required. It is of course essential that the HEP community has access to a myriad of spectrum generators, particularly those relevant to the Higgs sector, and more ideally several spectrum generators dedicated to the exact same model. This has been incredibly fruitful in studies based on the MSSM and NMSSM. This paper is an important step in that direction as it supplies a full two-loop spectrum generator, with the non-decoupled Dterm Higgs enhancements included in the model and in the self-energies for the calculation of the masses. What is particularly exciting and novel regarding our work is that our spectrum generator is on a par with all currently publicly available spectrum generators (such as [26,28-30]) and is the first dedicated spectrum generator to include the non decoupled Dterm enhancement to the Higgs sector. Secondly a dedicated study can address the important question of, if by removing the quiver of SU(3), one can then attempt to maximise the Higgs enhancement and achieve a linking field VEV, $v$, below $10 \mathrm{TeV}$, whilst keeping all gauge couplings perturbative to the Planck scale. In fact we found that this cannot be done. In addition this paper supplies a number of important and new results: it supplies a full derivation of the non-decoupled Dterm effect to all scalars and not just the Higgs fields, these contributions have so far gone unnoticed in the literature, but they may be quite relevant to precisions studies at $e^{+}, e^{-}$ colliders. Further, this implementation includes all RGEs to two loops for all parameters and all anomalous dimensions for all fields, supplying for the first time, the full anatomy of such a setup.

The outline of the paper is as follows: In Sect. 2 we give a first account of our key findings. In Sect. 3 we present the specific realisation of a quiver gauge theory that we will be studying in the following sections, discuss its general features and our choice of parameterisation of the soft term boundary conditions. Amusingly, these setups in some sense also model the effects of a truncated theory of gauge fields in an extra dimension and we make a small digression in Sect. 3.5 to discuss this connection. In Sect. 4 we discuss the concrete implementation of our quiver setup, the model's parameter 
space and the phenomenological constraints it is subject to. In Sect. 5 we present our results on the phenomenology of the model and discuss the features of its particle spectrum. Finally, in Sect. 6 we conclude. Appendix A contains details of the implementation of our framework along with some important relations. This paper is accompanied by a supplementary document containing details with regards to the implementation [27].

\section{Key results}

It is useful here to give a first summary of our key results. In this work we will show that

- With the setup we adopt it is perfectly possible to obtain a $125.5 \mathrm{GeV}$ Higgs with stops lighter than $2 \mathrm{TeV}$, gluinos of order $1600 \mathrm{GeV}$, light electroweakinos and sleptons potentially within the reach of the LHC, all within a GMSB framework (see benchmarks in Table 5).

- The correct Higgs mass is obtained through nondecoupled D-term contributions in the low-energy lagrangian that lift the Higgs boson mass at tree level, as shown in Fig. 4.

- These terms also modify the Higgs branching ratios but well within current LHC bounds, and could be probed by the ILC as seen in Fig. 6.

- The light uncoloured sparticle spectrum is achieved primarily due to the specific supersymmetry-breaking mediation mechanism we employ (see Fig. 7).

These results are obtained by implementing our model into the publicly available package SARAH, which enables us to create a spectrum generator in order to perform the RGE evolution of all model parameters at two loops, from the messenger scale $M$ down to the TeV scale, with GMSB boundary conditions.

We have implemented five gauge groups at full one- and two-loop running, plus one-loop self-energies, from the GUT or messenger scale, higgsing and breaking to the diagonal subgroup of four gauge groups, while finite shifts and threshold corrections are carefully applied for each degree of freedom.

We finally stress that we have implemented a conservative (precise) formulation of GMSB with full two-loop equations for soft masses in the hidden sector. Still at this level of specification a reasonably natural spectrum is obtained, which demonstrates the ease with which much lighter spectra would be obtainable if these high standards were relaxed or some more phenomenological parameterisation adopted. The framework that we have developed quite straightforwardly admits numerous extensions such as inclusion of $\mathrm{U}(1)$ kinetic mixing or quivering the $\mathrm{SU}(3)$ sector, tasks which are
Table 1 Gauge superfields of the model

\begin{tabular}{lllll}
\hline $\mathrm{SF}$ & Spin $\frac{1}{2}$ & $\mathrm{Spin} 1$ & $\mathrm{SU}(N)$ & Coupling \\
\hline$\hat{B}_{A}$ & $\tilde{B}_{A}$ & $B_{A}$ & $\mathrm{U}(1)_{A}$ & $g_{A 1}$ \\
$\hat{W}_{B}$ & $\tilde{W}_{B}$ & $W_{B}$ & $\mathrm{SU}(2)_{B}$ & $g_{B 2}$ \\
$\hat{g}$ & $\tilde{g}$ & $g$ & $\mathrm{SU}(3)_{c}$ & $g_{3}$ \\
$\hat{B}_{B}$ & $\tilde{B}_{B}$ & $B_{B}$ & $\mathrm{U}(1)_{B}$ & $g_{B 1}$ \\
$\hat{W}_{A}$ & $\tilde{W}_{A}$ & $W_{A}$ & $\mathrm{SU}(2)_{A}$ & $g_{A 2}$ \\
\hline
\end{tabular}

left for future work. All of these remarks will be clarified in the following sections.

\section{An electroweak quiver}

In this paper we wish to explore two different quiver models for comparison. The first carries the generic features of nondecoupled D-terms and, in the case of GMSB, suppressed scalar soft masses versus gauginos. The second is a flavourful extension of the first model to achieve lighter stops than the first two generations, which still obeys all anomaly cancellations. A common feature is that we will apply a gaugemediated supersymmetry-breaking scenario to both and both are characterised by the scale of supersymmetry breaking, $M$, and the VEVs of the linking fields ${ }^{2}, v$. In this section we outline these models and their features.

\subsection{The models and features}

Let us consider an electroweak two-site quiver with gauge group $G_{A} \times G_{B} \times \mathrm{SU}(3)_{c}$, where $G_{A}=\mathrm{SU}(2)_{A} \times \mathrm{U}(1)_{A}$ and $G_{B}=\mathrm{SU}(2)_{B} \times \mathrm{U}(1)_{B}$ as in Table 1 .

The two sites are connected by means of a pair of linking chiral superfields $\hat{L}, \hat{\tilde{L}}$. These superfields will play a crucial role both in the breaking of the enlarged gauge group to the MSSM gauge groups, by obtaining VEVs, and in the mediation of supersymmetry-breaking effects. Moreover, the setup includes a singlet chiral superfield $K$, whose role will be clarified shortly, as well as an additional superfield $A$ transforming as the adjoint of $\mathrm{SU}(2)_{B}$ that serves the role of giving masses to certain fermionic components of the linking fields upon $G_{A} \times G_{B}$ breaking. Much below the higgsing scale, $v$, the quiver fields usually decouple and so for phenomenological purposes at low energies the model is essentially MSSM-like with the addition of an effective action for the Higgs potential. It will be useful then to refer to the enlarged gauge groups as regime 1 and the MSSM as regime 2. This paper is based on two models which are as follows:

Model I: The first model (MI) is a basic example of a quiver model where the MSSM chiral superfields are taken to be

\footnotetext{
${ }^{2}$ Not to be confused with $v_{\text {ew }}$.
} 
Fig. 1 A picture to represent the quiver module of the electroweak sector for MI as in Table 2. The electroweak part of the supersymmetric standard model is on site A, with messenger fields $(\Phi, \tilde{\Phi})$ coupled to another site, site B. The linking fields $(L, \tilde{L})$ connect the two sites. The adjoint field $(A)$ is charged on the second site, site $\mathrm{B}$. The singlet field $(K)$ is not shown

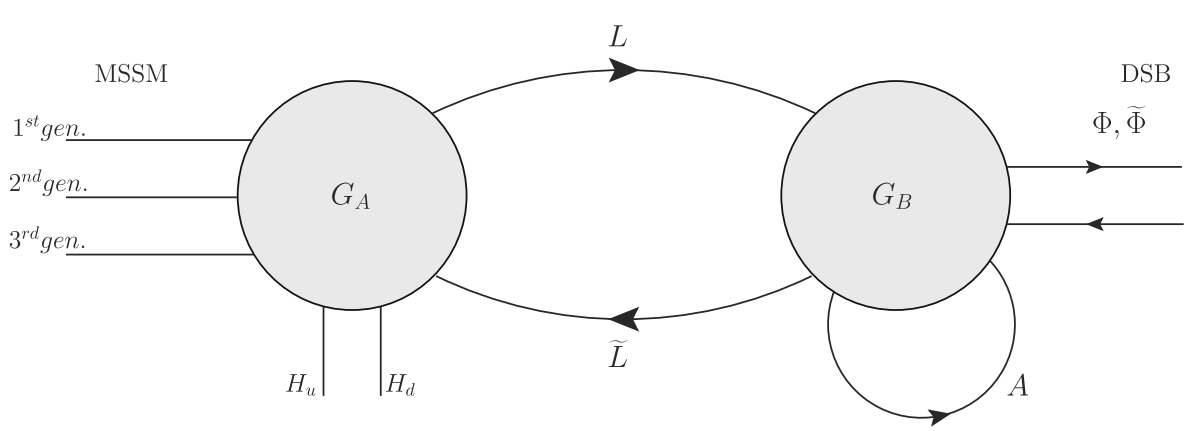

Table 2 Chiral superfields of the model MI. The index $f$ runs over all three generations. The representation ordering corresponds to $\left(\mathrm{SU}(2)_{A}, \mathrm{U}(1)_{A}, \mathrm{SU}(2)_{B}, \mathrm{U}(1)_{B}, \mathrm{SU}(3)_{c}\right)$. The superpotential is (3.1) and (3.2) and the supersymmetry-breaking messenger fields are charged under site B

\begin{tabular}{llll}
\hline $\mathrm{SF}$ & Spin 0 & Spin $\frac{1}{2}$ & $G_{A} \times G_{B} \times \mathrm{SU}(3)_{c} \quad(\mathrm{MI})$ \\
\hline$\hat{q}^{f}$ & $\tilde{q}^{f}$ & $q^{f}$ & $\left(\mathbf{2}, \frac{1}{6}, \mathbf{1}, 0, \mathbf{3}\right)$ \\
$\hat{l}^{f}$ & $\tilde{l}^{f}$ & $l^{f}$ & $\left(\mathbf{2},-\frac{1}{2}, \mathbf{1}, 0, \mathbf{1}\right)$ \\
$\hat{H}_{d}$ & $H_{d}$ & $\tilde{H}_{d}$ & $\left(\mathbf{2},-\frac{1}{2}, \mathbf{1}, 0, \mathbf{1}\right)$ \\
$\hat{H}_{u}$ & $H_{u}$ & $\tilde{H}_{u}$ & $\left(\mathbf{2}, \frac{1}{2}, \mathbf{1}, 0, \mathbf{1}\right)$ \\
$\hat{d}^{f}$ & $\tilde{d}_{R}^{f *}$ & $d_{R}^{f *}$ & $\left(\mathbf{1}, \frac{1}{3}, \mathbf{1}, 0, \overline{\mathbf{3}}\right)$ \\
$\hat{u}^{f}$ & $\tilde{u}_{R}^{f *}$ & $u_{R}^{f *}$ & $\left(\mathbf{1},-\frac{2}{3}, \mathbf{1}, 0, \overline{\mathbf{3}}\right)$ \\
$\hat{e}^{f}$ & $\tilde{e}_{R}^{f *}$ & $e_{R}^{f *}$ & $(\mathbf{1}, 1, \mathbf{1}, 0, \mathbf{1})$ \\
$\hat{L}$ & $L$ & $\psi_{L}$ & $\left(\mathbf{2},-\frac{1}{2}, \overline{\mathbf{2}}, \frac{1}{2}, \mathbf{1}\right)$ \\
$\hat{\tilde{L}}$ & $\tilde{L}$ & $\psi_{\tilde{L}}$ & $\left(\overline{\mathbf{2}}, \frac{1}{2}, \mathbf{2},-\frac{1}{2}, \mathbf{1}\right)$ \\
$\hat{K}$ & $K$ & $\psi_{K}$ & $(\mathbf{1}, 0, \mathbf{1}, 0, \mathbf{1})$ \\
$\hat{A}$ & $\mathrm{~A}$ & $\psi_{A}$ & $(\mathbf{1}, 0, \mathbf{3}, 0, \mathbf{1})$ \\
\hline
\end{tabular}

Boldface values refer to the dimension of the representation of nonabelian gauge groups whereas non-boldface ones to U(1) charges

charged under site A identically to their charges under the MSSM gauge group and neutral under site B (see Fig. 1 and Table 2). The superpotential of the MSSM-like matter is given by

$$
\begin{aligned}
W_{\mathrm{SSM}}= & Y_{u} \hat{u} \epsilon_{i j} \hat{q}^{i} \hat{H}_{u}^{j}-Y_{d} \hat{d} \epsilon_{i j} \hat{q}^{i} \hat{H}_{d}^{j} \\
& -Y_{e} \hat{e} \epsilon_{i j} \hat{l}^{i} \hat{H}_{d}^{j}+\mu \epsilon_{i j} \hat{H}_{u}^{i} \hat{H}_{d}^{j}
\end{aligned}
$$

with $i, j, k$ labelling $\mathrm{SU}(2)$ indices, and as this group is pseudo-real, the $\overline{2} \phi_{i}$ is simply $\epsilon_{i j} \phi^{j}$ of the 2 representation $\phi^{i}$ of $\mathrm{SU}(2)$. The superpotential of the quiver module is given by

$W_{\text {Quiver }}=\frac{Y_{K}}{2} \hat{K}\left(\hat{L}_{i}^{j} \hat{\tilde{L}}_{j}^{i}-V^{2}\right)+Y_{A} \hat{L}_{i}^{j} \hat{A}_{j}^{k} \hat{\tilde{L}}_{k}^{i}$.

A model with a similar structure albeit based on a more enlarged gauge group was first introduced in [7]. The general features of this model will be outlined below and unless stated in the text all RGEs and equations of this paper refer to MI.

Model II: The second model (MII) is a flavourful deformation of model I in that by construction the first and second generation MSSM chiral superfields are taken to be charged under site B and neutral under site A while the third generation and the Higgs fields are kept on site A (see Fig. 2 and Table 3). Similar representation assignments have been considered, for example, in [8,9] and then later in [13, 14,25] in the framework of models of natural supersymmetry that could potentially further address the flavour problem. The superpotential we use in regime 1 is

$$
\begin{aligned}
W_{\mathrm{MII}}= & Y_{u}^{3} \hat{u}^{3} \epsilon_{i j} \hat{q}^{3 i} \hat{H}_{u}^{j}-Y_{d}^{3} \hat{d}^{3} \epsilon_{i j} \hat{q}^{3 i} \hat{H}_{d}^{j} \\
& -Y_{e}^{3} \hat{e}^{3} \epsilon_{i j} \hat{l}^{3 i} \hat{H}_{d}^{j}+\mu \epsilon_{i j} \hat{H}_{u}^{i} \hat{H}_{d}^{j}
\end{aligned}
$$

plus the quiver superpotential (3.2). In regime 2 after returning to the MSSM gauge groups, we adopt the MSSM superpotential by (3.1).

\subsection{Gauge symmetry breaking}

We now describe certain features of the general setup. The superpotential (3.2) gives rise to a scalar potential which when minimised sets a vacuum expectation value for the scalar components of the linking fields of the model. Denoting

$L=\left(\begin{array}{ll}\varphi_{L 1} & \varphi_{L 2} \\ \varphi_{L 3} & \varphi_{L 4}\end{array}\right)$

in the absence of supersymmetry breaking, we write

$\langle L\rangle=\langle\tilde{L}\rangle=v \mathbb{I}_{2 \times 2}$ where $\operatorname{tr}\left(v^{2} \mathbb{I}_{2 \times 2}\right)=V^{2}$.

These break the gauge group $G_{A} \times G_{B}$ down to the diagonal subgroup $G_{\text {Diag }}=\mathrm{SU}(2)_{L} \times \mathrm{U}(1)_{Y}$, which are simply the MSSM gauge groups. The symmetry-breaking pattern takes the form

$$
\begin{aligned}
& \mathrm{SU}(2)_{A} \times \mathrm{SU}(2)_{B} \rightarrow \mathrm{SU}(2)_{L} \\
& \text { and } \mathrm{U}(1)_{A} \times \mathrm{U}(1)_{B} \rightarrow \mathrm{U}(1)_{Y} .
\end{aligned}
$$


Fig. 2 The quiver module of the electroweak sector for MII as in Table 3. The first and second generation matter is charged under site B and the third generation and MSSM Higgs fields are charged under site A. The messenger fields $(\Phi, \tilde{\Phi})$ are charged under site B. The linking fields $(L, \tilde{L})$ connect the two sites

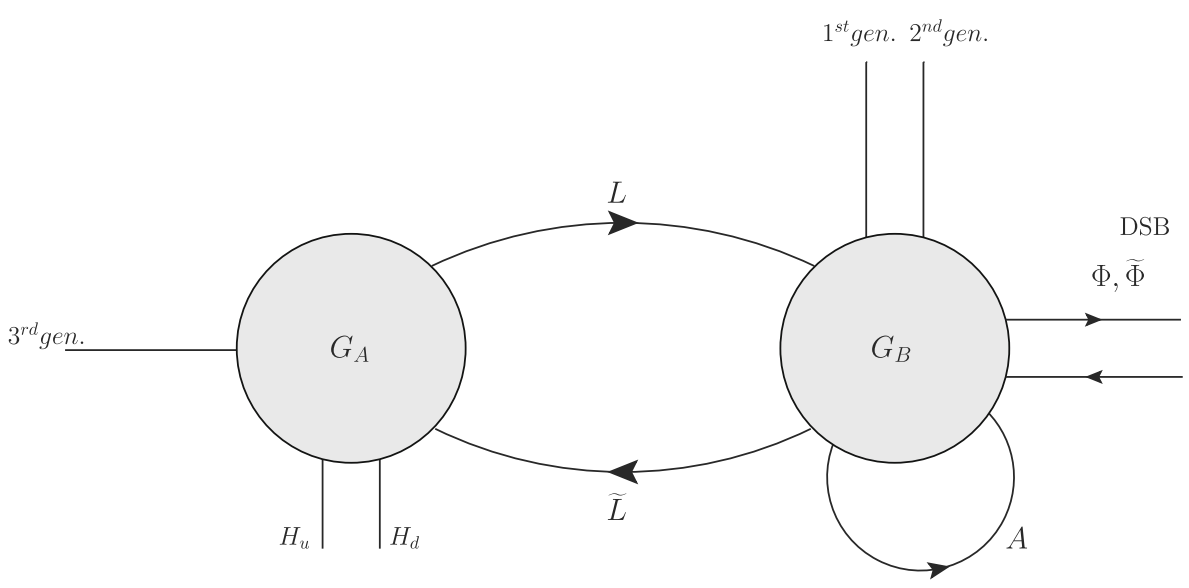

Table 3 Chiral superfields of the model MII. The first and second generation are charged under site $\mathrm{B}$. The third generation are charged under site $\mathrm{A}$. The representation ordering corresponds to $\left(\mathrm{SU}(2)_{A}, \mathrm{U}(1)_{A}, \mathrm{SU}(2)_{B}, \mathrm{U}(1)_{B}, \mathrm{SU}(3)_{c}\right)$. The superpotential is given by (3.3) and (3.2). The supersymmetry-breaking messenger fields are charged under site B

\begin{tabular}{llll}
\hline $\mathrm{SF}$ & Spin 0 & Spin $\frac{1}{2}$ & $G_{A} \times G_{B} \times \mathrm{SU}(3)_{c} \quad(\mathrm{MII})$ \\
\hline$\hat{q}^{1,2}$ & $\tilde{q}^{1,2}$ & $q^{1,2}$ & $\left(\mathbf{1}, 0, \mathbf{2}, \frac{1}{6}, \mathbf{3}\right)$ \\
$\hat{l}^{1,2}$ & $\tilde{l}^{1,2}$ & $l^{1,2}$ & $\left(\mathbf{1}, 0, \mathbf{2},-\frac{1}{2}, \mathbf{1}\right)$ \\
$\hat{d}^{1,2}$ & $\tilde{d}_{R}^{1,2 *}$ & $d_{R}^{1,2 *}$ & $\left(\mathbf{1}, 0, \mathbf{1}, \frac{1}{3}, \overline{\mathbf{3}}\right)$ \\
$\hat{u}^{1,2}$ & $\tilde{u}_{R}^{1,2 *}$ & $u_{R}^{1,2 *}$ & $\left(\mathbf{1}, 0, \mathbf{1},-\frac{2}{3}, \overline{\mathbf{3}}\right)$ \\
$\hat{e}^{1,2}$ & $\tilde{e}_{R}^{1,2 *}$ & $e_{R}^{1,2 *}$ & $(\mathbf{1}, 0, \mathbf{1}, 1, \mathbf{1})$ \\
$\hat{H}_{d}$ & $H_{d}$ & $\tilde{H}_{d}$ & $\left(\mathbf{2},-\frac{1}{2}, \mathbf{1}, 0, \mathbf{1}\right)$ \\
$\hat{H}_{u}$ & $H_{u}$ & $\tilde{H}_{u}$ & $\left(\mathbf{2}, \frac{1}{2}, \mathbf{1}, 0, \mathbf{1}\right)$ \\
$\hat{q}^{3}$ & $\tilde{q}^{3}$ & $q^{3}$ & $\left(\mathbf{2}, \frac{1}{6}, \mathbf{1}, 0, \mathbf{3}\right)$ \\
$\hat{l}^{3}$ & $\tilde{l}^{3}$ & $l^{3}$ & $\left(\mathbf{2},-\frac{1}{2}, \mathbf{1}, 0, \mathbf{1}\right)$ \\
$\hat{d}^{3}$ & $\tilde{d}_{R}^{3 *}$ & $d_{R}^{3 *}$ & $\left(\mathbf{1}, \frac{1}{3}, \mathbf{1}, 0, \overline{\mathbf{3}}\right)$ \\
$\hat{u}^{3}$ & $\tilde{u}_{R}^{3 *}$ & $u_{R}^{3 *}$ & $\left(\mathbf{1},-\frac{2}{3}, \mathbf{1}, 0, \overline{\mathbf{3}}\right)$ \\
$\hat{e}^{3}$ & $\tilde{e}_{R}^{3 *}$ & $e_{R}^{3 *}$ & $(\mathbf{1}, 1, \mathbf{1}, 0, \mathbf{1})$ \\
$\hat{L}$ & $L$ & $\psi_{L}$ & $\left(\mathbf{2},-\frac{1}{2}, \overline{\mathbf{2}}, \frac{1}{2}, \mathbf{1}\right)$ \\
$\hat{\tilde{L}}^{3 *}$ & $\tilde{L}_{\hat{K}}$ & $\psi_{\tilde{L}}$ & $\left(\overline{\mathbf{2}}, \frac{1}{2}, \mathbf{2},-\frac{1}{2}, \mathbf{1}\right)$ \\
$\hat{K}$ & $K$ & $\psi_{K}$ & $(\mathbf{1}, 0, \mathbf{1}, 0, \mathbf{1})$ \\
$\hat{A}$ & $\mathrm{~A}$ & $\psi_{A}$ & $(\mathbf{1}, 0, \mathbf{3}, 0, \mathbf{1})$ \\
\hline & & &
\end{tabular}

Now, including soft breaking masses $m_{L}^{2}, m_{\tilde{L}}^{2}$ for the linking fields, we expand the diagonal complex scalars into real scalar and pseudo-scalar components

$\varphi_{L 1,4}=v+i \sigma_{L 1,4}+\phi_{L 1,4}$

The $\sigma$ play the role of Goldstone bosons and get eaten by the gauge fields. Minimizing the scalar potential with respect to these fields leads to the tadpole equations, which at treelevel read $\frac{\partial V}{\partial \phi_{L 1}}=2 m_{L}^{2} v-\frac{1}{2} v Y_{k} \operatorname{Re}\left[Y_{k} V^{2}\right]+v^{3} Y_{k}^{2}$

and a similar expression for $\phi_{L 4}$. The value of the VEV $v$ can be obtained by requiring that the tadpoles should vanish. In practice it turns out to be much more convenient to take $v$ as an input parameter and compute the superpotential parameter $V^{2}$ from (3.8).

As a result of the quiver structure at different renormalisation scales $Q$ the following occur:

- Regime 1 is characterised by $M \geq Q>v$ with the full matter content and gauge groups of the quiver.

- In Regime 2, characterised by $v>Q$, the VEVs of the linking fields break the groups to the diagonal and the MSSM superfields transform under $G_{\text {Diag }} \equiv G_{\text {MSSM }}$ in the usual way.

- The $\mathrm{U}(1)$ gauge bosons $B_{A}, B_{B}$ mix to generate a massless and a massive state $B_{0}$ and $B_{m}$, the massless one being then the $\mathrm{U}(1)_{Y}$ boson and the $B_{m}$ being a heavy state. Similarly $W_{A}^{i}, W_{B}^{i}$ mix to form the massless $\mathrm{SU}(2)_{L} W_{0}^{i}$ gauge bosons as well as three heavy states $W_{m}^{i}$ with the corresponding mixing angles discussed below. The masses of the heavy gauge bosons are simply given by

$$
m_{v, i}^{2}=2\left(g_{A, i}^{2}+g_{B, i}^{2}\right) v^{2} .
$$

In this setup we have not considered the quiver structure for $\mathrm{SU}(3)$. Whilst this is mostly due to practical reasons, given the difficulty of a full and proper implementation of a higgsed SU(3) as in $[10,14]$, it is also not necessary for our purposes. Indeed naively we sacrifice a GUT completion, but clearly we are expecting our model to be valid only up to the messenger scale in the case of GMSB and anyway it should be quite straightforward to tidy up this setup to restore gauge unification without sacrificing the key results of this work. In this sense our setup is both minimal enough, and 
yet concrete enough to be a "theoretical simplified model" which captures relevant features of a much larger range of possibilities; for example, it incorporates an extra SU(2) and $\mathrm{U}(1)$.

\subsection{Supersymmetry-breaking and soft breaking terms for gauge mediation}

In principle the models above may be combined with any supersymmetry breaking scenario, for example mSUGRA, or some more phenomenological parameterisation. The model contains a large number of soft terms. The soft breaking scalar potential reads

$$
\begin{aligned}
& \mathcal{V}_{\text {soft }}=B_{\mu} \epsilon_{i j} H_{u}^{i} H_{d}^{j}+T_{d}^{a b} H_{d}^{i} \tilde{d}_{a} \tilde{q}_{b i}+T_{e}^{a b} H_{d}^{i} \tilde{e}_{a} \tilde{l}_{b i} \\
& +T_{u}^{a b} H_{u}^{i} \tilde{u}_{a} \tilde{q}_{b i}+\frac{1}{2} L_{V^{2}} K+T_{A} L_{j}^{i} A_{k}^{j} \tilde{L}_{i}^{k}+T_{K} K L_{j}^{i} \tilde{L}_{i}^{j} \\
& +m_{I J}^{2} \phi_{I}^{*} \phi_{J}+m_{A}^{2}|A|^{2}+\frac{1}{2} m_{L}^{2}\left(|L|^{2}+|\tilde{L}|^{2}\right)+m_{K}^{2}|K|^{2} .
\end{aligned}
$$

$a, b$ are flavour indices and $i, j, k \mathrm{SU}(2)$ indices, which are lowered with $\epsilon_{i j}$. The soft terms for the fermions are

$$
\begin{aligned}
\mathcal{L}_{\text {soft }} \supset & \frac{1}{2}\left(m_{\tilde{g}} \tilde{g} \tilde{g}+m_{\tilde{B}_{B}} \tilde{B}_{B} \tilde{B}_{B}+m_{\tilde{W}_{B}} \tilde{W}_{B} \tilde{W}_{B}\right. \\
& \left.+m_{\tilde{B}_{A}} \tilde{B}_{A} \tilde{B}_{A}+m_{\tilde{W}_{A}} \tilde{W}_{A} \tilde{W}_{A}\right)+ \text { h.c. }
\end{aligned}
$$

In what follows, the RGE evolution of all parameters in this scalar potential will be accounted for at two loops.

In this work we focus on gauge mediation and take the highest scale of the RGE evolution to be $M$, the characteristic mass scale of supersymmetry breaking which in perturbative models is the messenger scale. As is typical of these perturbative gauge-mediated supersymmetry-breaking scenarios, we model the supersymmetry-breaking sector with a set of messenger fields coupled to a spurion. Such a messenger sector can (and should) be generalised [12], although we will conform to this standard paradigm. The superpotential we use is of the form

$W_{\text {Messenger }}=X \Phi \tilde{\Phi}$

where $X$ is a spurion with $X=M+\theta^{2} F$ and $\Phi, \tilde{\Phi}$ are representative of fundamental and antifundamental messenger fields, respectively, charged under $\mathrm{SU}(3)_{C}$ and $\mathrm{SU}(2)_{B}$, $\mathrm{U}(1)_{B}$, but not under the A-site electroweak group. This leads to a scale $\Lambda=F / M$ which may differ for each gauge group so we can in general write $\Lambda_{1,2,3}$ for the three gauge groups. The messenger fields and spurion are integrated out at $M$ to generate the soft terms, the explicit equations for which are supplied below. ${ }^{3}$

Here we describe the gauge mediation parameterisation of the above soft terms.

\subsubsection{The trilinear, bilinear and linear terms}

The trilinear T-terms (or A-terms) are taken to be zero at the messenger scale, including those corresponding to $Y_{K}$ and $Y_{A}$. The linear soft term $L_{V^{2}}$ for the singlet $\phi_{K}$ is also taken to be vanishing at the messenger scale. In GMSB, the bilinear term $B_{\mu}$ is expected to be zero at the supersymmetrybreaking scale and should be generated by RG running. In what follows we will use the standard SUSY Les Houches Accord GMSB conventions [26,28-33] according to which tadpole equations are solved for $\mu$ and $B_{\mu}$ and $\tan \beta$ is given as an input. Above the scale of $G_{A} \times G_{B}$ breaking, the $\beta$ function for $B_{\mu}$ reads at one loop

$$
\begin{aligned}
\beta_{B \mu}^{(1)}= & \left(-\frac{3}{5} g_{A 1}^{2}-3 g_{A 2}^{2}+\operatorname{Tr}\left(Y_{d}^{\dagger} Y_{d}\right)\right. \\
& \left.+\operatorname{Tr}\left(Y_{e}^{\dagger} Y_{e}\right)+\operatorname{Tr}\left(Y_{u}^{\dagger} Y_{u}\right)\right) B_{\mu} \\
& +\frac{2}{5}\left(3 g_{A 1}^{2} m_{\tilde{B}_{A}}+15 g_{A 2}^{2} m_{\tilde{W}_{A}}\right. \\
& \left.+5 \operatorname{Tr}\left(Y_{d}^{\dagger} T_{d}\right)+5 \operatorname{Tr}\left(Y_{e}^{\dagger} T_{e}\right)+5 \operatorname{Tr}\left(Y_{u}^{\dagger} T_{u}\right)\right) \mu
\end{aligned}
$$

which results in a large $B_{\mu}$ if the $g_{A i}$ are relatively large. The equations are similar below the quiver breaking scale. Note that there are also two-loop contributions in both regimes.

\subsubsection{Gaugino soft masses}

For $\mathrm{SU}(3)_{c} \times \mathrm{SU}(2)_{B} \times \mathrm{U}(1)_{B}$ the gauginos acquire soft masses according to the standard GMSB formula

$m_{\lambda, r}=N \Lambda\left(\frac{g_{r}^{2}}{16 \pi^{2}}\right) g(x)$

where $x=F / M^{2}, \Lambda=F / M$ and $r$ refers to the corresponding gauge group. $N=\left(n_{5 \text { plets }}+3 n_{10 \text { plets }}\right)$ is the messenger index and the function $g(x)$ is the standard function appearing in GMSB gaugino soft masses.

As the messenger sector is not charged under $\mathrm{U}(1)_{A}$ and $\mathrm{SU}(2)_{A}$, the corresponding gauginos are taken to be massless at the messenger scale:

$m_{\tilde{B}_{A}}=m_{\tilde{W}_{A}} \equiv 0$.

\footnotetext{
${ }^{3}$ It would be interesting to extend this work to include explicit messenger fields and run supersymmetrically from the GUT scale to the messenger scale, include messenger effects at the scale $\mathrm{M}$ and then run down to the electroweak scale.
} 
One might imagine that such a feature could be detrimental to the low-energy spectrum. However, the mass matrices of the gauginos are rather complicated including supersymmetric Dirac masses as well as the above soft terms, so this turns out not to be the case: the mass eigenstates result from a combination of the site $A$ and site $B$ gauginos ${ }^{4}$. The Majorana soft masses of the broken theory can be found by identifying the masses of the relevant components of the mixing matrices, at the threshold scale $\mathcal{O}(v)$ (see also Appendix A.1). As the A-site gauginos do not obtain significant soft masses until the scale $v$, the RGEs of matter charged under site A will not feel these effects until a scale $Q<v$. This turns to be advantageous for naturalness as now the threshold scale $T_{\text {scale }}=v$ acts as a cutoff to the leading RGE logarithm. Such an effect will become especially important for an $\mathrm{SU}(3)_{A} \times \mathrm{SU}(3)_{B}$ quiver, as then the A-site gaugino would only influence the RGEs between $M_{e w}$ and $T_{\text {scale }}$ and have essentially no effect on the RGEs of site A matter above this scale.

\subsubsection{Site A scalar soft masses}

The scalar soft masses depend on the site under which the corresponding superfields are actually charged, relative to those of the messenger fields. We will always take the messenger fields to be on site B.

Fields charged under $G_{A}$ get soft masses at two loops from mediation along the quiver

$m_{A}^{2}=N \sum_{i=1,2} 2 \Lambda_{i}^{2} C_{i}(r)\left(\frac{g_{i}^{2}}{16 \pi^{2}}\right)^{2} s\left(x, y_{i}\right)$,

where $y=m_{v} / M$ with $m_{v}$ being the heavy gauge boson mass, $M$ the messenger scale and $g_{i}$ is the corresponding coupling constant. The quadratic Casimir invariants $C_{i}(r)$ are $C_{1}(Y)=3 / 5 Y^{2}$ for fields charged under $\mathrm{U}(1)$ with hypercharge $Y$ and $C_{2}(2)=3 / 4$ for doublets under $\mathrm{SU}(2)$. In MI, where all MSSM chiral superfields reside in site A, this formula serves as a boundary condition for all electroweak contributions to the scalar soft masses. In MII, this formula only applies to the third generation sfermions and the Higgs scalars, whereas the first two generation sfermions receive their soft masses according to (3.18).

The form of the function $s(x, y)$, associated with gauge mediation along a two-site quiver, can be found in [12,23,24] and is given in both analytical and graphical form in Appendix A.5. By inspection we can see how the mediation of supersymmetry breaking along the quiver has the effect of reducing the site A scalar soft masses with respect

\footnotetext{
4 Full details of this matrix and all mass matrices as well as all RGEs and tadpole equations may be found in the supplementary material accompanying the arXiv version of our paper or by interfacing through Mathematica with the SARAH model file.
}

to usual gauge-mediated supersymmetry breaking. In particular, $s(x, y)$ has the limit

$s(x, \infty)=f(x)$

where $f(x)$ is the usual GMSB formula. This formula interpolates between $y \rightarrow \infty$ of GMSB and the suppressed scalar regime as $y \rightarrow 0$, where scalars get their leading soft mass at three loops from additional contributions, which arise anyway from RG evolution.

\subsubsection{Site B scalar soft masses}

Scalar fields charged under $G_{B}$ and $\mathrm{SU}(3)_{c}$ receive standard GMSB soft masses according to

$m_{B}^{2}=N \sum_{i=1,2,3} 2 \Lambda_{i}^{2} C_{i}(r)\left(\frac{g_{B i}^{2}}{16 \pi^{2}}\right)^{2} f(x)$.

The quadratic Casimir invariants are $C_{1}\left(Y_{B}\right)=3 / 5 Y_{B}^{2}$ for fields with charge $Y_{B}$ under $\mathrm{U}(1)_{B}$ whereas $C_{2}(2)=3 / 4$ for the linking fields $L$ and $C_{2}(3)=2$ for the $\mathrm{SU}(2)_{B}$ adjoint $A$ field. All coloured scalars in our setup are SU(3) triplets, for which $C_{3}(3)=4 / 3$. Note that in the case of site A coloured scalars, the full soft mass is given by the sum of (3.16) and the third term of (3.18).

\subsubsection{The singlet scalar $K$ soft mass}

The superfield $K$ is a gauge singlet and its scalar soft mass is vanishing at the messenger scale,

$m_{K}^{2}=0$.

It evolves a positive value through (7.29) and so does not pose a phenomenological issue, but if one did wish to assign a tree-level soft mass, two approaches are possible: it may be interesting to consider that it is not a singlet under some other group or that it couples directly to messenger fields through a term of the form $K \Phi \tilde{\Phi}$. In the latter case it can develop a one-loop soft mass.

\subsubsection{Linking scalar soft masses}

The linking fields formally get their soft masses from applying (3.18) to describe the soft terms for $m_{L}^{2}$ and $m_{\tilde{L}}^{2}$. We will however not be using this formula in order to compute the linking field soft masses. Instead, we will promote them to free parameters of the model. The reasons for this choice will be clarified in the following section. To be noted is that in this setup the two linking field masses are equal, $m_{L}^{2}=m_{\tilde{L}}^{2}$. 


\subsection{Linking to the MSSM}

Below the quiver breaking scale the gauge group and particle content of the model are those of the minimal supersymmetric standard model with gauge groups $\mathrm{SU}(3)_{c} \times \mathrm{SU}(2)_{L} \times \mathrm{U}(1)_{Y}$. The gauge couplings between the unbroken and the broken theory are matched as

$$
\frac{1}{g_{i}^{2}}=\frac{1}{g_{A i}^{2}}+\frac{1}{g_{B i}^{2}} \Leftrightarrow g_{i}^{2}=\frac{g_{A i}^{2} g_{B i}^{2}}{g_{A i}^{2}+g_{B i}^{2}},
$$

with $i=1,2$ for $\mathrm{U}(1)_{Y} \times \mathrm{SU}(2)_{L}$. If one of the two gauge couplings is strong, the other should be weak. Then at low energies the diagonal or MSSM gauge coupling will be of the order of whichever is weaker. This is a key feature which allows for these models to lift the tree-level Higgs mass whilst being consistent with perturbative unification. The various gauge couplings of the model are simply related through two rotation angles ${ }^{5}$

$\cos \theta_{i}=\frac{g_{i}}{g_{A i}}, \quad \sin \theta_{i}=\frac{g_{i}}{g_{B i}}$.

In Appendix A.4 we present additional comments on threshold effects that enter the coupling constants and other parameters calculation below the quiver breaking scale. The angles $\theta_{1}, \theta_{2}$ are free parameters of our setup. Varying these amounts to changing the relative strengths between each site and we typically choose the A-sites to be stronger to enhance additional contributions to the Higgs mass, as we will explain below.

One of the most interesting features of this class of models is that non-decoupled D-terms may arise [3,34] in the lowenergy lagrangian. The real uneaten scalar components of the linking fields appear in both the A- and B-site scalar Dterm potential and when integrated out generate an effective action which includes the terms

$$
\begin{aligned}
\delta \mathcal{L}= & -\frac{g_{1}^{2} \Delta_{1}}{8}\left(H_{u}^{\dagger} H_{u}-H_{d}^{\dagger} H_{d}\right)^{2} \\
& -\frac{g_{2}^{2} \Delta_{2}}{8} \sum_{a}\left(H_{u}^{\dagger} \sigma^{a} H_{u}+H_{d}^{\dagger} \sigma^{a} H_{d}\right)^{2},
\end{aligned}
$$

where

$$
\Delta_{1}=\left(\frac{g_{A 1}^{2}}{g_{B 1}^{2}}\right) \frac{m_{L}^{2}}{m_{v 1}^{2}+m_{L}^{2}}, \quad \Delta_{2}=\left(\frac{g_{A 2}^{2}}{g_{B 2}^{2}}\right) \frac{m_{L}^{2}}{m_{v 2}^{2}+m_{L}^{2}} .
$$

\footnotetext{
${ }^{5}$ Here we should stress an important notation subtlety. In all relations applying to the messenger scale as well as in all RGE expressions, the U(1) coupling constants are taken to be SU(5) GUT-normalised, so for example $g_{1}=g_{1, \mathrm{GUT}}=\sqrt{5 / 3} g^{\prime}$, with $g^{\prime}$ being the usual Standard Model hypercharge coupling constant. In all other relations, the GUT normalisation is dropped and $g_{1}$ identical to $g^{\prime}$. This is done in order to follow the SARAH package conventions.
}

It is particularly informative to see how in this class of models, these terms can work to lift the Higgs mass without large radiative corrections. In the MSSM, the one-loop Higgs mass in the limit $m_{A^{0}} \gg m_{Z}$ can be written as [35]

$$
\begin{aligned}
m_{h, 1}^{2} \simeq & m_{z}^{2} \cos ^{2} 2 \beta+\frac{3}{4 \pi^{2}} \frac{m_{t}^{4}}{v_{e w}^{2}} \\
& \times\left[\ln \frac{M_{S}^{2}}{m_{t}^{2}}+\frac{X_{t}^{2}}{M_{S}^{2}}\left(1-\frac{X_{t}^{2}}{12 M_{S}^{2}}\right)\right]
\end{aligned}
$$

where $M_{S}^{2}=m_{\tilde{t}_{1}} m_{\tilde{t}_{2}}$ for $m_{\tilde{t}_{1}}, m_{\tilde{t}_{2}}$ as defined in Appendix A.1, and $v_{e w}$ is the electroweak Higgs VEV, such that the upper limit on the tree-level Higgs mass $\left(m_{h, 0}\right)$ is set by the $\mathrm{Z}$ boson mass $m_{z}$. This expression assumes that the left- and right-handed soft masses of the stops are equal. Note that $X_{t}=A_{t}-\mu \cot \beta$, and for convenience the sfermion mixing matrices are provided explicitly in Eq. (7.12) of Appendix 1. In our case however, there may in principle be a sizeable shift to the Higgs mass at tree level, $m_{h, 0}$, which takes the precise form

$m_{h, 0}^{2}=\left[m_{z}^{2}+\left(\frac{g_{1}^{2} \Delta_{1}+g_{2}^{2} \Delta_{2}}{2}\right) v_{e w}^{2}\right] \cos ^{2} 2 \beta$.

Arguably this enhancement is favoured over that of the NMSSM for a simple reason: in the NMSSM typically

$m_{h, 0}^{2}=m_{z}^{2} \cos 2 \beta+\lambda^{2} v_{e w}^{2} \sin 2 \beta$

where $\lambda$ is the coupling between the Higgs singlet and doublet fields appearing in the superpotential term $\lambda S H_{u} H_{d}$. This creates a tension between wanting a large $\tan \beta$ to enhance the first term, and a small $\tan \beta$ for the second, forcing one to accept very large values of $\lambda$. As a result $\lambda$ ends up nonperturbative before the GUT scale.

It is the above observation that forms the basis for the construction of natural spectra in the class of models that we examine: the Higgs mass can now be substantially increased already at tree level when these new contributions become large. Of course this enhancement is completely independent of the method by which supersymmetry breaking effects are transmitted to the MSSM. We have simply chosen GMSB in this paper on the one hand to demonstrate that it is still a natural candidate for supersymmetry-breaking mediation, and on the other hand because in our electroweak GMSB quiver the sleptons can be naturally lighter than their coloured counterparts. This potential enhancement of the tree-level Higgs mass is only significant in certain areas of the model's full parameter space. Concretely, for this contribution to be sizeable we must have $g_{A i}^{2} / g_{B i}^{2} \geq 1$ and $m_{L} \sim \mathcal{O}\left(m_{v, i}\right)$.

However, this mechanism introduces some additional finetuning to the theory, since the Higgs mass now receives an additional quadratically divergent contribution at one loop, induced by the linking fields and cut off by $m_{L}^{2}$. This additional fine-tuning should be kept under control in order to 
not counterbalance the improved naturalness of the model with respect to traditional mGMSB. An estimate of the maximal size of $m_{L}$ can be found following the arguments of [5]: demanding less than $10 \%$ additional fine-tuning approximately bounds

$$
\frac{g_{\mathrm{SM}}^{2} \Delta}{16 \pi^{2}} \frac{m_{L}^{2}}{m_{h}^{2}}<10 .
$$

Requiring, for example, $\Delta=0.2$ in (3.23) allows $m_{L}^{2}$ in the $10^{6}-10^{8} \mathrm{GeV}^{2}$ range and sets an upper value $v<10^{5} \mathrm{GeV}$ so that the additional D-terms do not decouple. So in summary we would ideally want $v<10^{5} \mathrm{GeV}$ and $m_{L}<$ $10 \mathrm{TeV}$. Note that $v$ is also bounded from below both by electroweak precision tests and direct searches for new gauge bosons.

The non-decoupled D-terms also appear in the tadpole equations

$$
\begin{gathered}
\frac{\partial V}{\partial \phi_{d}}=\frac{1}{8}\left(-8 v_{u} \operatorname{Re}\left[B_{\mu}\right]+\left(g_{1}^{2}+g_{2}^{2}+g_{1}^{2} \Delta_{1}^{2}+g_{2}^{2} \Delta_{2}^{2}\right) v_{d}^{3}\right. \\
\left.+v_{d}\left[8 m_{H_{d}}^{2}+8|\mu|^{2}-\left(g_{1}^{2}+g_{2}^{2}+g_{1}^{2} \Delta_{1}^{2}+g_{2}^{2} \Delta_{2}^{2}\right) v_{u}^{2}\right]\right)
\end{gathered}
$$$$
\begin{aligned}
& \frac{\partial V}{\partial \phi_{u}}=\frac{1}{8}\left(-8 v_{d} \operatorname{Re}\left[B_{\mu}\right]+8 v_{u}|\mu|^{2}+v_{u}\right. \\
& \left.\quad \times\left[8 m_{H_{u}}^{2}-\left(g_{1}^{2}+g_{2}^{2}+g_{1}^{2} \Delta_{1}^{2}+g_{2}^{2} \Delta_{2}^{2}\right)\left(-v_{u}^{2}+v_{d}^{2}\right)\right]\right)
\end{aligned}
$$

modifying the vacuum structure, as well as the Higgs mixing matrices, which may be found in Appendix A.2.

Additional soft mass terms for all scalars appear in regime 2 of the model at effective one loop, from integrating out the heavy gauge and linking fields [10]

$$
\begin{aligned}
\delta m_{\tilde{f}}^{2}= & \sum_{i}\left(\frac{g_{i}}{4 \pi}\right)^{2} C_{k}^{\tilde{f}}\left[m_{v_{i}}^{2} \tan ^{2} \theta_{i} \log \left(1+\frac{2 m_{L}^{2}}{m_{v_{i}}^{2}}\right)\right. \\
& \left.+2 \sin ^{2} \theta_{i}\left(1-3 \sin ^{2} \theta_{i}\right) m_{i, B}^{2}\right],
\end{aligned}
$$

which are also implemented into the model and importantly the soft mass parameters are matched across the threshold scale. $^{6}$

\subsection{An extra dimensional digression}

Quiver models are naturally related to extra dimensional setups through deconstruction. For early ideas on the topic we refer the interested reader to [36]. The contemporary formulation of the topic was initiated in [6,37-39], whereas for recent work relating to $\mathcal{N}=1$ see for instance $[12,24,40]$.

${ }^{6}$ There are further three-loop terms if the SU(3) sector is quivered.
It should therefore be expected that these non-decoupled Dterms, (3.23), have a natural interpretation in terms of extra dimensional models. We swiftly sketch and motivate this relationship, which certainly warrants further study on its own. The quiver construction may be related to $\mathcal{N}=1$ super Yang-Mills (or $\mathcal{N}=2$ [41-43]) in five dimensions [44], which contains a vector multiplet and chiral adjoint $V+\Phi$. Suppose we compactify on four flat dimensions times a small interval of length $R$. The scalar component of $\Phi=\left(\Sigma+i A_{5}\right)$, and in analogy to the quiver, $A_{5}$ plays the role of the Goldstone bosons and these are eaten to generate the KaluzaKlein masses such that we may identify $1 / R \sim v$ of (3.7). To obtain the non-decoupled D-terms we write the lagrangian in the off-shell formulation

$\mathcal{L}_{5}=\frac{1}{2} D^{2}+D\left(\partial_{5} \Sigma\right)-\frac{1}{2} \partial_{\mu} \Phi \partial^{\mu} \Phi^{*}+m_{\text {soft }}^{2} \Sigma \Sigma+\cdots$

The ellipses denote not just the rest of the bulk action but also any terms generated on bulks and boundaries that may be of use such as the boundary terms

$$
\int_{\partial \mathcal{M}}(\Sigma D+\cdots) \text {. }
$$

To see how this action may generate a non-decoupled D-term we define $\mathcal{H}=\left(H_{u}^{\dagger} H_{u}-H_{d}^{\dagger} H_{d}\right)$. Then there may be bulk or boundary terms of the usual form

$\mathcal{L} \supset \frac{1}{2} \mathcal{H} D$

Integrating out the auxiliary scalar field $D$ gives rise to the D-term scalar potential. The field $\Sigma$, the real uneaten scalar degrees of freedom, corresponds to the real uneaten degrees of freedom in the linking fields $L, \tilde{L}$ of the quiver. It is this field $\Sigma$, when integrated out which generates the non decoupled D-term (3.23). In such a scenario, the $m_{v}$ of the quiver is related to the Kaluza-Klein mass scale $m_{k k}$ which is $O(1 / R)$, the effective length scale of the extra dimension. As such, for these terms to be of relevance $\pi / R \leq m_{\text {soft }}$. We hope to return to this topic in a further publication, but for now we effectively model this feature with quiver models as they are a more controlled environment which are more amenable to spectrum generators. It is certainly interesting to speculate that as our model has a $v$ of $\mathcal{O}\left(10^{4}\right) \mathrm{GeV}$, that this corresponds to an "effective" extra dimensional length scale of roughly $\mathcal{O}\left(10^{-18}\right) \mathrm{cm}$.

\section{Tools and observables}

In order to study the low-energy phenomenology of our setup in a consistent manner, it is necessary to perform the RGE 
evolution of all couplings and mass parameters from the highest energy scale of the theory down to the $\mathrm{TeV}$ scale, properly imposing all boundary conditions. Here we describe the construction of a tailor-made spectrum generator for the quiver model. We further discuss the parameter space we adopt as well as the constraints it is subject to.

\subsection{Implementing a quiver framework for phenomenological studies}

In order to perform the RGE evolution of the models' parameters and masses and compute the resulting low-energy particle spectra, we implemented the two model variants into the publicly available Mathematica package SARAH 3.3 [17-19]. SARAH is a "spectrum generator generator", which includes a library of models that may communicate with HEP tools that are widely used in most phenomenological studies $[30,33,45-54]$. In particular, SARAH performs the task of generating Fortran routines compatible with the SPheno spectrum generator [18].

In order to implement our model, we have used the possibility offered by the package to implement and link two different "regimes". These regimes correspond to those introduced in Sect. 3.2, each being characterised by a set of gauge groups, a particle content and a superpotential that need to be specified. Regime 1 includes, for both models MI and MII, the full $G_{A} \times G_{B} \times \mathrm{SU}(3)_{c}$ gauge group along with the full quiver particle content, while the superpotential is given by Eqs. (3.1) and (3.2) for MI and by Eqs. (3.3) and (3.2) for MII. In both cases in regime 2 we have the MSSM, which we supplement with an effective action to account for and study the effects of the non-decoupled D-terms, which are of crucial importance, as discussed in Sect. 3.4. These terms are properly included in all loop calculations, self-energies, branching ratios and vertices of regime 2 . We would have preferred to implement our model using a single regime such that these terms would be automatically generated, and as there are other terms for other fields, however, we found it was not practical to integrate out so many fields in full. Moreover, it was preferable to include an additional regime with the MSSM gauge and matter configuration to ease communication of SPheno with packages such as HiggsBounds, used to check the compatibility of the model with experimental constraints as discussed below.

In addition to these ingredients, we need to specify on the one hand boundary conditions for all soft parameters at the messenger scale $M$ and on the other hand matching conditions for the parameters of the two regimes at the $G_{A} \times G_{B}$ breaking scale which we typically take to coincide with $v$, the linking field VEV. The boundary conditions are applied according to the discussion and relations given in Sect. 3.3, while the matching conditions follow the lines described in Sect. 3.4.
The renormalisation group equations for both regimes are then calculated by SARAH at two loops and appropriate Fortran routines are generated that can then be taken over by SPheno to perform the numerical analysis. Concretely, the implementation includes full one- and two-loop RGEs for five gauge groups, mixing matrices for all fields in the quiver and the MSSM including associating Goldstones with massive gauge bosons and gauge fixings, full two-loop RGEs for the VEV of the linking fields themselves, two-loop RGEs for $B \mu$, loop-level solutions for the tadpole equations, oneand two-loop anomalous dimensions for all fields and twoloop RGEs for all soft breaking parameters, linear, bilinear and trilinear. The MSSM particle masses are computed at one loop, however, the full two-loop corrections to the Higgs mass are implemented in SPheno following the calculation in Refs. [55-58].

All in all, the RGE evolution is described by three energy scales

$M_{\text {messenger }} \longrightarrow T_{\text {scale }} \longrightarrow M_{\text {ew }}$.

Following standard practice, the highest energy scale of the theory is taken to be the messenger scale $M$, where all boundary conditions resulting from the quiver structure, including exact formulae for GMSB soft masses have been implemented and imposed. Again as usual, the running ends at the electroweak scale, which is used as an input scale for the MSSM parameters. The intermediate mass scale $T_{\text {scale }}$ is associated with the quiver breaking scale as it separates the two regimes, and at this scale appropriate matching boundary conditions are applied including finite shifts that result from integrating out the heavy fields of the theory (see Appendix A.4). We choose it to be equal to the VEV of the linking fields, $T_{\text {scale }}=v$. All soft terms of regime 2 are matched to regime 1 as described in Sect. 3.4 and for the soft masses of the winos and binos in Appendix A.1. Note that the gluino finite shifts are also accounted for as given in Appendix A.4.

It is important to stress that the high-scale boundary conditions themselves may be seen as being separable from the model (the matter content, gauge groups and superpotential) and may be changed with ease, if one wished to explore, for example, different supersymmetry-breaking scenarios.

The implementation detailed above, i.e. the construction of a tailor-made spectrum generator for our model, allows us to create a model file for SPheno, which further permits us to study a quiver model in a complete manner, as we can study the influence of RG effects of all the gauge groups and matter content in the highest regime to the low-energy spectrum, at the two-loop order. On the practical level, this model can also serve as a first step for the implementation of more complete or complicated setups, such as a model including an additional SU(3). It would further be trivial to change the representation assignments of the Higgs fields in 
order to study chiral non-decoupled D-terms [59] or flavour models, with the same precision. However, we point out that the implementation of the electroweak only quiver studied here leads to an interesting phenomenology in its own right, allowing for naturally (although moderately) heavier squarks relative to sleptons.

\subsection{Parameter space and constraints}

It is now useful to describe the process through which we choose our parameter space and the regions we will study in the next section. We, moreover, describe some preliminary findings that could be of interest for model-building purposes.

\subsubsection{Choosing the parameter space}

The electroweak quiver we consider can be described by a basic set of six parameters

$M, \Lambda, v, \tan \beta, \theta_{1}, \theta_{2}$,

where $M$ is the messenger scale, the SUSY-breaking scale $\Lambda=F / M, F$ being the SUSY-breaking F-term, $\tan \beta$ is the Higgs vev ratio and $\theta_{1}, \theta_{2}$ are the mixing angles between sites $A$ and $B$ for $U(1)$ and $S U(2)$, respectively.

As an initial step, we performed extended scans over large regions of parameter space for MI, imposing the full set of GMSB boundary conditions described in Sect. 3.3, including the exact relations (3.18) for the linking field soft masses. We focussed in particular on regions where $v \lesssim 40$ $\mathrm{TeV}$, where according to the discussion of Sect. 3.4 the nondecoupled D-terms should be most efficient in lifting the treelevel Higgs boson mass. A first finding of these searches is that we could not find viable points when the linking field VEV was much below $10 \mathrm{TeV}$, as often here either the Asite couplings become non-perturbative before the messenger scale, the electroweak vacuum becomes unstable or the RGE code simply would not converge for the numerical precision requirements imposed (a relative error of $0.5 \%$ ). Where none of these issues occur, the values of $\Lambda$ are typically low and close to $v$, such that the linking fields soft masses $m_{L}$ are too low for the D-terms to have an important impact on the Higgs mass.

Motivated by the perturbativity issues, we implemented MII where we expect that by removing some of the matter fields from site A the RGE running will be reduced [14]. We found that although the situation does improve, it still seems to be quite difficult to achieve substantial contributions to the Higgs tree-level mass from the non-decoupled D-terms due to the fact that $m_{L}^{2}$ is again driven too low.

These results lead us to slightly enlarge our parameter space by promoting the linking field squared soft mass $m_{L}^{2}$ to be a free parameter instead of being given by (3.18). This is interesting from a theoretical perspective, as it motivates pursuing models that might provide the additional contribution to $m_{L}^{2}$ needed in order to achieve a substantial D-term contribution to the Higgs mass. For example, it would be interesting to study whether this can be realised in extensions to the model including an additional $\mathrm{SU}(3)$ or $\mathrm{U}(1)$ kinetic mixing. Within the scope of our work, the choice to make $m_{L}^{2}$ free can be seen as a phenomenological parametrisation of the linking field soft masses along the lines of similar choices made in many supersymmetry-breaking mediation schemes.

With this small modification, we find that it is indeed perfectly possible to achieve the required D-term size in order to reproduce the observed Higgs mass while keeping the stop masses well below $2 \mathrm{TeV}$. Furthermore as expected, the mediation of SUSY-breaking along the quiver acts as a suppression mechanism for the uncoloured sparticle masses, yielding electroweakinos and sleptons lying roughly in the range $[400,1000] \mathrm{GeV}$, which is on the boundary of being within the LHC reach $[60,61]$. At this point, due to the differing bounds on coloured and non-coloured sparticles at the LHC, we introduce a second modification to the original setup that consists of dissociating the scale $\Lambda_{3}$ from $\Lambda_{1,2}$. Note, however, that this is a minor modification as the two scales will not differ by orders of magnitude but only by $\mathcal{O}(1)$ multiplicative factors. We will see that this setup allows for a rich phenomenology with interesting features.

\subsubsection{Constraints}

We carried out extensive scans of the parameter space described in the previous paragraph within generous intervals. We are interested in areas of parameter space which are characterised by low values of $v$ and moderate splittings between $v$ and $m_{L}$, such that the additional D-terms do not decouple from the low-energy theory and the uncoloured scalars are light. In what follows, we will therefore present results that concern a subregion of the parameter space that meets a series of requirements.

First, we wish to obtain a Higgs mass lying in the range $[122.5,128.5] \mathrm{GeV}$. This interval envelops on the one hand the experimental uncertainty in the Higgs mass measurement $[1,2,62]$, while being sufficiently generous to account for uncertainties in the theoretical mass spectrum determination $[63,64]^{7}$. For naturalness reasons, we require this value for the Higgs mass to be achieved for stop masses as low as possible. The stop mass is governed by $\Lambda_{3}$, which also controls the masses of the lower generation squarks and the gluino. Strong exclusion limits on these masses arise from ATLAS and CMS null searches for jets plus missing energy, e.g. $m_{\tilde{g}}>1600 \mathrm{GeV}$ for $m_{\tilde{q}_{1,2}}>2000 \mathrm{GeV}[66,67]$. We

\footnotetext{
7 Throughout our calculations we assume a constant moderate top quark mass of $m_{t}=173 \mathrm{GeV}$ [65].
} 
are guided by these bounds in choosing a lower limit for $\Lambda_{3}$. Note that in this work, we choose not to quantify the amount of fine-tuning for each point of the models we study, which constitutes a work in its own right involving numerous subtleties (see for example the recent discussion in [68]). It is, however, at least clear that qualitatively, having stops lighter than benchmark minimal GMSB improves the relative naturalness of the model, and this motivates our choice of upper limit on $\Lambda_{3}$.

At the same time, according to the comments made in Sect. 3.4, we should avoid reintroducing excessive finetuning via the non-decoupled D-terms. Moreover, in order for the setup to be realistic, we must satisfy the condition $m_{L}^{2}<m_{v}^{2}$, but we should not approach the limit $m_{L}^{2} \ll m_{v}^{2}$ where the quiver-induced $\mathrm{D}$-terms decouple. These requirements lead to choose $m_{L}^{2}$ within the range $\left[10^{7}, 10^{8}\right] \mathrm{GeV}^{2}$. Also note that as mentioned above, for very low values of $v$ SPheno faces convergence issues. The parameter $\Lambda_{1,2}$ is mainly subject to constraints from searches for charged sleptons and charginos at LEP, i.e. $92 \mathrm{GeV}$ for charginos degenerate with the lightest neutralino, and $103.5 \mathrm{GeV}$ otherwise [69]. Lower limits on sleptons staus and sneutrinos of 68 and $51 \mathrm{GeV}$, respectively, were also obtained at LEP [70-72]. Finally, given that the non-decoupled D-terms contribute a shift to the Higgs mass as $m_{Z}$ does, i.e. with a factor $\cos 2 \beta$ (3.25), as opposed to the factor $\sin 2 \beta$ in the $\operatorname{NMSSM~(3.26),~}$ we explore a rather standard MSSM-like range for $\tan \beta$.

From our numerical analysis we find that this set of requirements is satisfied by adopting the following parameter value ranges:

$$
\begin{aligned}
& 2.1 \times 10^{5} \mathrm{GeV} \leq M \leq 3.0 \times 10^{5} \mathrm{GeV}, \\
& 4.0 \times 10^{4} \mathrm{GeV} \leq \Lambda_{1,2} \leq 1.9 \times 10^{5} \mathrm{GeV}, \\
& 1.9 \times 10^{5} \mathrm{GeV} \leq \Lambda_{3} \leq 2.1 \times 10^{5} \mathrm{GeV}, \\
& 1 \times 10^{7} \mathrm{GeV}^{2} \leq m_{L}^{2} \leq 1 \times 10^{8} \mathrm{GeV}^{2}, \\
& 1.5 \times 10^{4} \mathrm{GeV} \leq v \leq 4 \times 10^{4} \mathrm{GeV}, \\
& 5 \leq \tan \beta \leq 30, \\
& 0.8 \leq \theta_{1}, \theta_{2} \leq 1.4 .
\end{aligned}
$$

We have, moreover, chosen $\operatorname{sign} \mu=+1$, a low value for the messenger index, $N=1$, and a fixed common value for the $A$ and $K$ field Yukawa couplings $Y_{A}=Y_{K}=0.8$.

Apart from the theoretical and experimental constraints so far mentioned, the low-energy spectrum is subject to further bounds. In the Higgs sector, in addition to obtaining the lightest Higgs boson mass within the observed region, we must ensure that its properties and decay modes comply to current LHC observations. As an example, it is well known that enhancing the Higgs mass through non-decoupled D-terms enhances simultaneously the Higgs boson couplings to down-type quarks $[5,15,59]$. In order to test whether the Higgs sector is compatible with the con-
Table 4 Low-energy observable constraints imposed in our analysis

\begin{tabular}{ll}
\hline Observable & Accepted range \\
\hline$B_{s} \rightarrow X_{s} \gamma$ & {$[2.78,4.32] \times 10^{-4}[74]$} \\
$\delta a_{\mu}$ & $<20 \times 10^{-10}[65]$ \\
$\Delta \rho$ & $<1.2 \times 10^{-3}[65]$ \\
$B R\left(B_{s} \rightarrow \mu^{+} \mu^{-}\right)$ & $<7.7 \times 10^{-9}[75]$ \\
\hline
\end{tabular}

straints coming from the LHC and the TeVatron, we have linked SPheno to HiggsBounds-4.0.0 [51]. Taking our analysis a step further, we have also linked SPheno to HiggsSignals-1.0.0 [73], which allows us to test in particular whether the lightest Higgs boson properties are in agreement with all relevant existing mass and signal strength measurements from the LHC and TeVatron.

Finally, we use the built-in functionalities of SPheno in order to apply a set of necessary low-energy constraints, all of which are taken at $3 \sigma$ : the SUSY contributions to the muon anomalous magnetic moment $\delta a_{\mu}$ and the branching ratios $B R\left(B_{s} \rightarrow X_{s} \gamma\right)$ and $B R\left(B_{s} \rightarrow \mu^{+} \mu^{-}\right)$and, due to the presence of relatively light sfermions in our spectra, the $\rho$ parameter. The allowed ranges used for these observables are shown in Table 4, where theoretical uncertainties and experimental errors are added in quadrature.

\section{Results}

Having described our model, and how it is implemented in SARAH, we turn to the study of the low scale spectrum, which we find has several interesting features. Examples of complementary representative points, one for MI and two for MII, are given in Table 5.

In Table 5 we observe that particles of the electroweak sector can be substantially lighter than those of the coloured sector. This arises due to the quiver structure of the model, as explained in Sect. 3.3, which provides a suppression factor $s(x, y)$ for the non-coloured scalar masses, for details see Appendix A.5. The suppression is further enhanced by the fact that we have chosen to study the range of parameter space where $\Lambda_{1,2}<\Lambda_{3}$. Therefore it is possible for the masses of electroweakinos, sleptons and the heavy Higgs bosons to lie well below $1 \mathrm{TeV}$. One observes in Table 5 that this results in the next-to-lightest supersymmetric particles (NLSPs), being either a neutralino, as in MI and MIIb, or stau, as in MIIa. A sneutrino NLSP is also possible as will be discussed in detail later in this section. As we only consider a single SU(3) gauge group at the high scale, the masses of the coloured sparticles do not experience this suppression. This means that the coloured sector lies in general between 1.5 and $2.5 \mathrm{TeV}$, the stops being the lightest squarks. However, in MII, a splitting is generated between the left and right stop soft masses, for reasons discussed below, as shown in point 
Table 5 Mass spectra of three example points for MI and MII, along with the associated input parameters as defined in Sect. 4.2. Note that $m_{\tilde{f}_{12, L / R}}$ and $m_{\tilde{f}_{12, L / R}}$ are the masses of the lower and third generation left/right-handed squarks and sleptons, and that the sneutrino and sbottom masses can be inferred via $m_{\tilde{v}_{i}} \sim m_{\tilde{f}_{i, L}}, m_{\tilde{b}_{L}} \sim m_{\tilde{t}_{L}}$

\begin{tabular}{|c|c|c|c|}
\hline & MI & MIIa & MIIb \\
\hline \multicolumn{4}{|c|}{ Input values } \\
\hline M & $233 \mathrm{TeV}$ & $288 \mathrm{TeV}$ & $260 \mathrm{TeV}$ \\
\hline$\Lambda_{1,2}$ & $44.9 \mathrm{TeV}$ & $85.6 \mathrm{TeV}$ & $111 \mathrm{TeV}$ \\
\hline$\Lambda_{3}$ & $190 \mathrm{TeV}$ & $206 \mathrm{TeV}$ & $208 \mathrm{TeV}$ \\
\hline$m_{L}^{2}$ & $47.3 \mathrm{TeV}^{2}$ & $83.3 \mathrm{TeV}^{2}$ & 86.2 $\mathrm{TeV}^{2}$ \\
\hline$v$ & $26.2 \mathrm{TeV}$ & $26.5 \mathrm{TeV}$ & $25.4 \mathrm{TeV}$ \\
\hline$\theta_{1}, \theta_{2}$ & $1.18,1.13$ & $1.09,1.33$ & $1.05,1.04$ \\
\hline $\tan \beta$ & 16 & 12 & 28 \\
\hline \multicolumn{4}{|c|}{ Squark sector } \\
\hline$m_{\tilde{t}_{1}}$ & $1.84 \mathrm{TeV}$ & $1.99 \mathrm{TeV}$ & $409 \mathrm{GeV}$ \\
\hline$m_{\tilde{t}_{2}}$ & $1.98 \mathrm{TeV}$ & $2.06 \mathrm{TeV}$ & $3.49 \mathrm{TeV}$ \\
\hline$A_{\mathrm{t}}$ & $-442 \mathrm{GeV}$ & $-146 \mathrm{GeV}$ & $-141 \mathrm{GeV}$ \\
\hline$m_{\tilde{b}_{R}}$ & $1.95 \mathrm{TeV}$ & $2.05 \mathrm{TeV}$ & $2.56 \mathrm{TeV}$ \\
\hline$m_{\tilde{q}_{12, L}}$ & $2.05 \mathrm{TeV}$ & $2.12 \mathrm{TeV}$ & $2.19 \mathrm{TeV}$ \\
\hline$m_{\tilde{q}_{12, R}}$ & $1.97 \mathrm{TeV}$ & $2.10 \mathrm{TeV}$ & $2.14 \mathrm{TeV}$ \\
\hline \multicolumn{4}{|c|}{ Slepton sector } \\
\hline$m_{\tilde{l}_{12, L}}$ & $738 \mathrm{GeV}$ & $314 \mathrm{GeV}$ & $515 \mathrm{GeV}$ \\
\hline$m_{\tilde{l}_{3, L}}$ & $736 \mathrm{GeV}$ & $315 \mathrm{GeV}$ & $440 \mathrm{GeV}$ \\
\hline$m_{\tilde{l}_{12, R}}$ & $901 \mathrm{GeV}$ & $183 \mathrm{GeV}$ & $262 \mathrm{GeV}$ \\
\hline$m_{\tilde{l}_{3, R}}$ & $899 \mathrm{GeV}$ & $110 \mathrm{GeV}$ & $4.31 \mathrm{TeV}$ \\
\hline \multicolumn{4}{|c|}{ Gaugino sector } \\
\hline$m_{\tilde{\chi}_{1}^{0}}$ & $53.2 \mathrm{GeV}$ & $116 \mathrm{GeV}$ & $154 \mathrm{GeV}$ \\
\hline$m_{\tilde{\chi}_{2}^{0}}$ & $99.3 \mathrm{GeV}$ & $242 \mathrm{GeV}$ & $306 \mathrm{GeV}$ \\
\hline$m_{\tilde{\chi}_{3}^{0}}$ & $187 \mathrm{GeV}$ & $750 \mathrm{GeV}$ & $818 \mathrm{GeV}$ \\
\hline$m_{\tilde{\chi}_{4}^{0}}$ & $222 \mathrm{GeV}$ & $755 \mathrm{GeV}$ & $823 \mathrm{GeV}$ \\
\hline$m_{\tilde{\chi}_{1}^{ \pm}}$ & $96.8 \mathrm{GeV}$ & $242 \mathrm{GeV}$ & $306 \mathrm{GeV}$ \\
\hline$m_{\tilde{\chi}_{2}^{ \pm}}$ & $225 \mathrm{GeV}$ & $756 \mathrm{GeV}$ & $823 \mathrm{GeV}$ \\
\hline$m_{\tilde{g}}$ & $1.62 \mathrm{TeV}$ & $1.66 \mathrm{TeV}$ & $1.75 \mathrm{TeV}$ \\
\hline \multicolumn{4}{|c|}{ Higgs sector } \\
\hline$m_{h_{0}}$ & $125 \mathrm{GeV}$ & $127 \mathrm{GeV}$ & $125 \mathrm{GeV}$ \\
\hline$m_{H_{0}}$ & $720 \mathrm{GeV}$ & $792 \mathrm{GeV}$ & $885 \mathrm{GeV}$ \\
\hline$m_{A_{0}}$ & $721 \mathrm{GeV}$ & $796 \mathrm{GeV}$ & $894 \mathrm{GeV}$ \\
\hline$m_{H_{ \pm}}$ & $726 \mathrm{GeV}$ & $799 \mathrm{GeV}$ & $893 \mathrm{GeV}$ \\
\hline
\end{tabular}

MIIb of Table 5. In the following we will study the spectra of these models in terms of their compatibility with the current experimental constraints described in the previous section and the prospects for detecting signs of $\mathrm{TeV}$-scale sparticles in the near future.

\subsection{The Higgs mass and couplings}

As the non-decoupled D-terms lift the tree-level Higgs mass, as in Eq. (3.25), here we investigate the range of stop masses in these models for which $m_{h}$ lies in the desired range, and how the stop contribution compares to that of the nondecoupled D-terms. The same non-decoupled D-terms can affect the couplings of the Higgs, so we further investigate these couplings in light of current and future experimental measurements.

\subsubsection{The Higgs mass}

As mentioned in Sect. 4.2, we have chosen $\Lambda_{3}$ such that the masses of gluino and the first and second generation squarks lie above the LHC exclusion limits. In MI, this translates into the stop masses being close to $2 \mathrm{TeV}$, which means that the shift in the tree-level Higgs mass required in order to obtain $m_{h} \sim 125.5 \mathrm{GeV}$ is small, and the required soft mass of the linking field remains below $10 \mathrm{TeV}$. The situation is fairly similar in MII, however, we find that there is a slight tendency for a splitting to arise between the left- and right-handed stop soft masses, due to the RGEs driving the left-handed mass downwards, and the right-handed mass upwards. This can be understood in terms of the differences between the RGE equations for the two models, where in MII above the quiverbreaking scale the Higgs soft masses are only affected by the third generation squarks, whereas for MI the Higgs soft mass RGE equations contain all generations. This results in a larger splitting between the up- and down-type Higgs soft masses which further generates a larger splitting between the left and right-handed stop. The distribution of the masses of the light and heavy stops i.e. $m_{\tilde{t}_{1}}$ and $m_{\tilde{t}_{2}}$ as defined in Appendix A.1 for the two models are displayed in Fig. 3. Here the allowed points are shown in yellow and those points excluded by the various constraints described in Sect. 4.2 in grey. We clearly observe that for MII a larger splitting between the stops is possible, and the lighter stop may be as light as $400 \mathrm{GeV}$, as seen in the benchmark point MIIb in Table 5.

In Fig. 4 we have plotted the Higgs mass as a function of $\tan \beta$ for the two variants of our model. Here the bright red points respect $m_{\tilde{t}_{1}}<2 \mathrm{TeV}$ and all constraints imposed, the pale red points only comply with the low-energy constraints and the grey points are excluded. The full two-loop corrections to the Higgs mass are implemented in SPheno following the calculation in Refs. [55-58]. We conclude that a Higgs mass within the limits $\sim 125.5 \pm 3.0 \mathrm{GeV}$ is achievable in both MI and MII. Note that the larger range in $m_{h}$ for MII can be explained by the larger range in stop masses. Indeed, when the left- and right-handed stop soft masses are not equal, as shown in Fig. 3 for MII, the simplified expression for the one-loop Higgs mass given in Eq. (3.24) is no longer valid. An additional correction must be added to Eq. (3.24) of the form [76]

$$
\Delta m_{h, 1}^{2}=\frac{3 m_{Z}^{2}}{16 \pi^{2} v_{e w}^{2}}\left(1-\frac{8}{3} \sin \theta_{W}^{2}\right) \cos 2 \beta m_{t}^{2} \ln \left(\frac{m_{\tilde{q}_{L}^{3}}^{2}}{m_{\tilde{u}_{R}^{3}}^{3}}\right),
$$



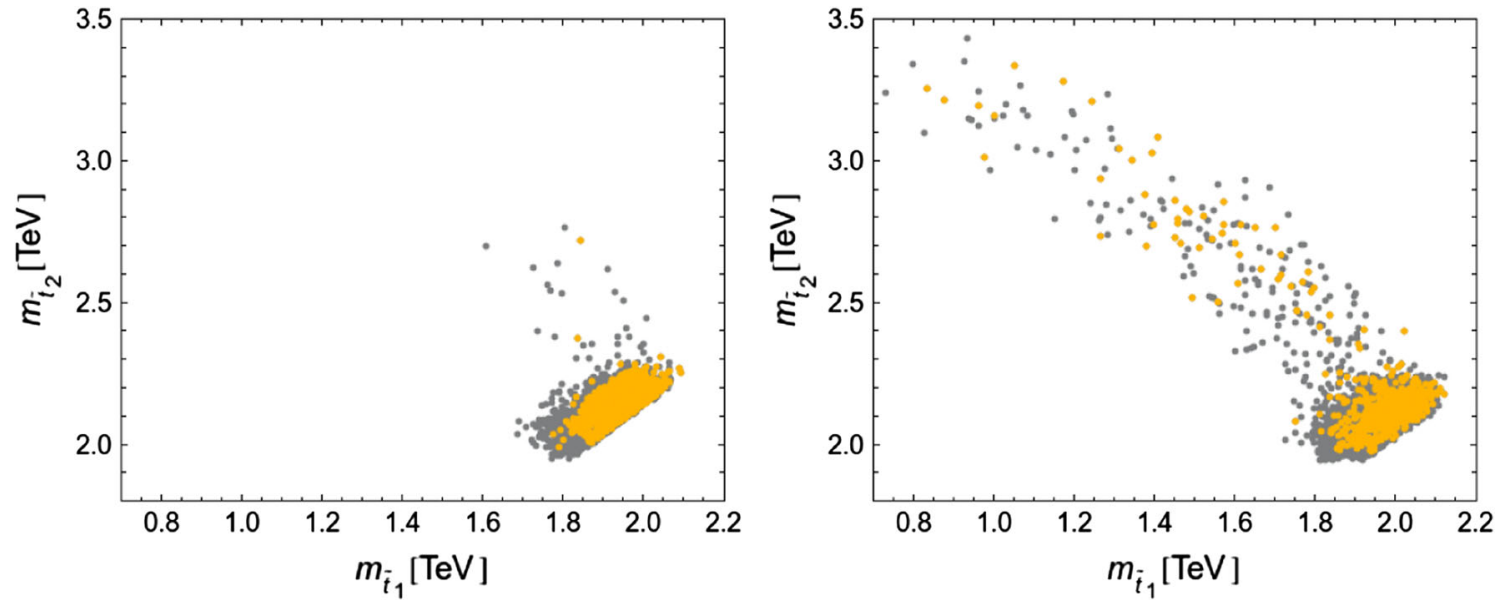

Fig. 3 The mass of the heavy stop $m_{\tilde{t}_{2}}$ as a function of $m_{\tilde{t}_{1}}$ for MI (left $)$ and MII (right). Points satisfying low-energy and the Higgs mass constraints are shown in yellow, whereas the remaining excluded points are shown in grey

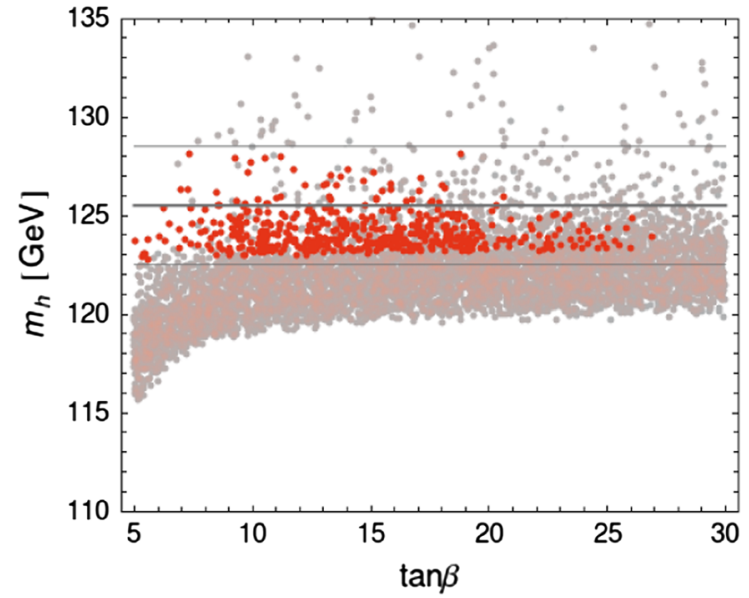

Fig. 4 The Higgs mass as a function of $\tan \beta$ for MI (left) and MII (right). Points satisfying all constraints and $m_{\tilde{t}_{1}}<2 \mathrm{TeV}$ are bright red, whereas paler points indicate that stop masses are in the range

which for the case $m_{\tilde{q}_{L}^{3}}^{2}<m_{\tilde{u}_{R}^{3}}$ in MII induces an enhancement to the Higgs mass of around 1-2 GeV. Note that the sfermion mixing matrix is defined in Appendix A.2. As the bright red points correspond to $m_{\tilde{t}_{1}}<2 \mathrm{TeV}$, this further demonstrates that the effect of the non-decoupled D-term seems to reduce the fine-tuning by allowing lighter stops than in standard GMSB.

To make the distinction between the non-decoupled Dterm and radiative, i.e. stop sector, contributions to the Higgs mass clearer, we compare the tree-level result $m_{h, 0}$ to the full two-loop result $m_{h, 2}$ in Fig. 5. Here the bright blue points respect $m_{\tilde{t}_{1}}<2 \mathrm{TeV}$ and all constraints imposed, the pale blue points only satisfy low-energy restrictions and the grey points are excluded. As opposed to the mGMSB result, where $m_{h, 0}$ is bounded by $m_{Z}$, here we observe that a shift of up to $10 \mathrm{GeV}$ is possible for both MI and MII, while keeping

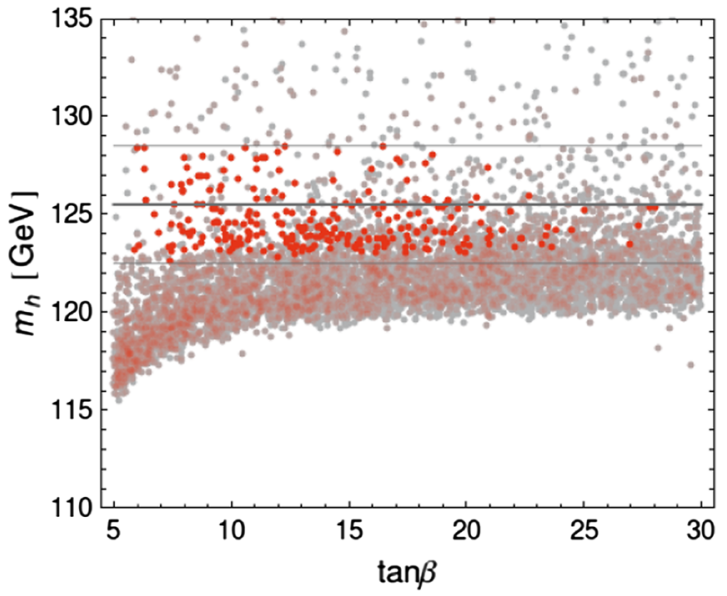

$2 \mathrm{TeV}<m_{\tilde{t}_{1}}<2.3 \mathrm{TeV}$ and only low-energy constraints are satisfied. Grey points are excluded. The thick (thin) grey lines denote the central value (uncertainty) on $m_{h}$

$m_{L}<10 \mathrm{TeV}$. This in turn means that the contribution of the radiative corrections required to achieve $m_{h} \sim 125.5 \mathrm{GeV}$ is diminished, rendering the model more natural. Interestingly, the splitting of the stops observed in MII results in a distinct difference between the two plots in Fig. 5, which can be understood from Eq. (5.1). Despite the fact that the range in $\Lambda_{3}$ is the same for both MI and MII, the stop splitting enhances the size of the radiative corrections, resulting in a smaller shift in the tree-level Higgs mass required to obtain a value of $m_{h}$ in agreement with experiment.

\subsubsection{The Higgs couplings}

Since the discovery of the Higgs boson, not only has its mass been used to discriminate between supersymmetric models but also its couplings; see e.g. $[5,77,78]$. The deviation of 


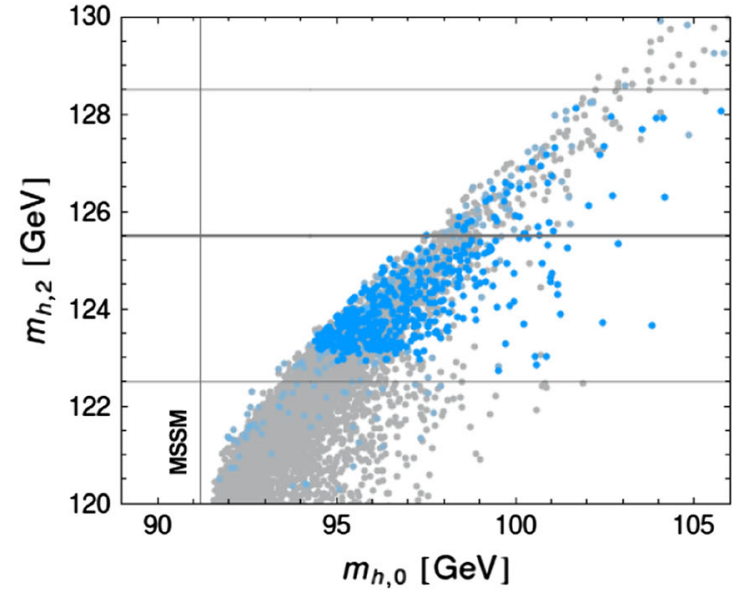

Fig. 5 The two-loop Higgs mass $\left(m_{h, 2}\right)$ as a function of the tree-level Higgs mass $\left(m_{h, 0}\right)$ for MI (left) and MII (right). Points satisfying all constraints for which $m_{\tilde{t}_{1}}<2 \mathrm{TeV}$ are bright blue, paler points indicate low-energy constraints are satisfied and stop masses are in the range 2

these couplings from the SM can be parameterised via the set of ratios $r_{i}$, for $i=b, \gamma, g$ etc., where

$r_{i}=\frac{\Gamma_{\mathrm{MSSM}}(h \rightarrow i i)}{\Gamma_{\mathrm{SM}}(h \rightarrow i i)}$.

The $r_{i}$ are further related to the signal strengths $\mu_{i}$ normally quoted by ATLAS and CMS; see e.g. Refs. [79,80]. Note that the errors on the measured signal strengths are still too large to make detailed interpretations about the potential underlying SUSY model, and at present the $\mu_{i}$ are all SM compatible, and therefore we will not tackle a precise calculation of the various signal strengths in this work. As mentioned above, we, however, make sure that the lightest Higgs boson signal strengths are in agreement with the existing LHC and TeVatron measurements within $3 \sigma$, employing the HiggsSignals code. Recent studies by both ATLAS and CMS $[60,61]$ have found that with a luminosity of $300 \mathrm{fb}^{-1}$ at the $14 \mathrm{TeV}$ LHC, an uncertainty on the measurement of $r_{b}$ should only be 10-13\%. A more sensitive determination, however, should be possible at the international linear collider (ILC), where for a centre of mass energy $(\sqrt{s})$ of $500 \mathrm{GeV}, 500 \mathrm{fb}^{-1}$ and polarised beams $P\left(e^{+}, e^{-}\right)=(-0.8,+0.3)$, a precision of $1.8 \%$ is quoted in Ref. [81].

In our model, the non-decoupled D-terms result in a tree-level contribution to the Higgs coupling to down-type fermions. The ratio $r_{b}\left(=r_{\tau}\right.$ at tree level $)$ takes the form

$r_{b}=-\frac{\sin \alpha}{\cos \beta}$

where $\alpha$ is the angle between the two Higgs doublets in the MSSM, and is defined by [14]

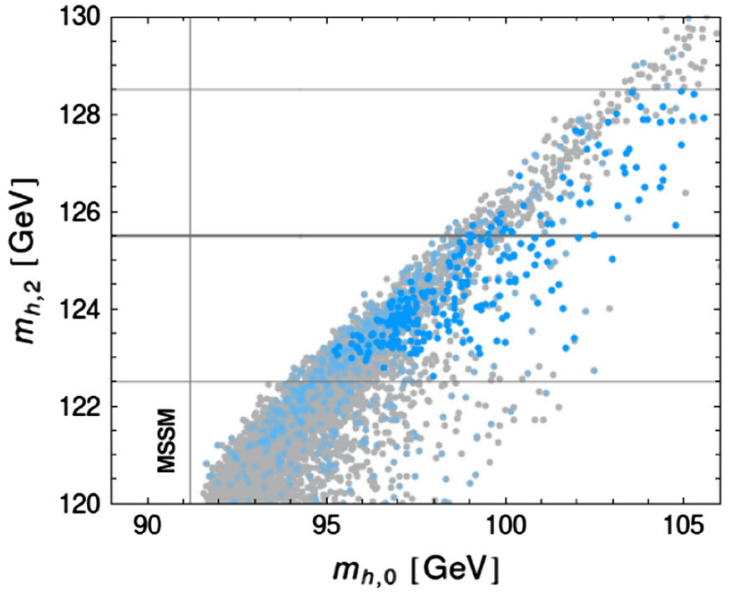

$\mathrm{TeV}<m_{\tilde{t}_{1}}<2.3 \mathrm{TeV}$, and points excluded by low-energy constraints are shown in grey. The vertical line indicates the MSSM bound on $m_{h, 0}$. The thick (thin) grey lines denote the central value (uncertainty) on $m_{h}$

$\tan 2 \alpha=\frac{m_{A_{0}}^{2} \cos 2 \beta+m_{h, 0}^{2}}{m_{A_{0}}^{2} \cos 2 \beta-m_{h, 0}^{2}} \tan 2 \beta$.

Here $m_{A_{0}}$ is the pseudo-scalar Higgs mass of the MSSM and $m_{h, 0}$ is the tree-level Higgs mass given in Eq. (3.25). We plot the Higgs mass as a function of $r_{b}$ in Fig. 6, where again the bright red points respect $m_{\tilde{t}_{1}}<2 \mathrm{TeV}$ and all constraints imposed, the pale red points only satisfy low-energy constraints (i.e. they do not comply with our requirements in the Higgs sector) and the grey points are excluded. We find that only a $\sim 2 \%$ change in $r_{b / \tau}$ is required for MI, and a $\sim 4 \%$ change for MII in order to obtain a Higgs mass of $125.5 \mathrm{GeV}$, with $m_{\tilde{t}_{1}}<2 \mathrm{TeV}$. Note that in our model the enhanced coupling to down-type fermions results in a suppression of the signal strength $\mu_{\gamma}[5,59]$, which was not favoured by initial measurements at the LHC [82]. However, as data has collected, the results for $\mu_{\gamma}$ appear more and more SM-like $[79,80]$. As in this work the tree-level shift in the Higgs mass only needs to be under $10 \mathrm{GeV}$, we consider small values of $\Delta_{1,2}$, for which the deviation in the coupling of the Higgs to down-type sfermions are well within the current LHC bounds (see e.g. Ref. [79]) as shown in Fig. 6. Such deviations should start to become detectable at the $\sqrt{s}=500 \mathrm{GeV}$ ILC.

\subsection{Sparticle searches at the LHC}

As mentioned in Sect. 4.2, the choice of $\Lambda_{3}$ ensures that the masses of the gluino and lower generation squarks approximately respect the limits from direct searches at the LHC. On the other hand, as the scale of the electroweak sector is set by $\Lambda_{1,2}$, by allowing $\Lambda_{1,2}<\Lambda_{3}$ we explore a range of parameter space for which the electroweak sector has a greater chance of being observed at the LHC. Further, as 


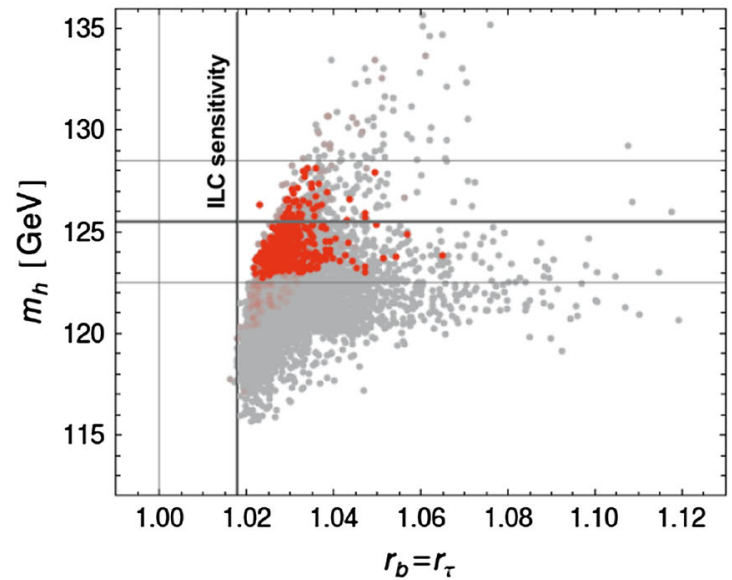

Fig. 6 The Higgs mass as a function of $r_{b}=r_{\tau}$ for MI (left) and MII (right). Points satisfying all constraints and $m_{\tilde{t}_{1}}<2 \mathrm{TeV}$ are bright red, whereas paler points indicate that stop masses are in the range $2 \mathrm{TeV}<m_{\tilde{t}_{1}}<2.3 \mathrm{TeV}$ and only low-energy constraints are satisfied.

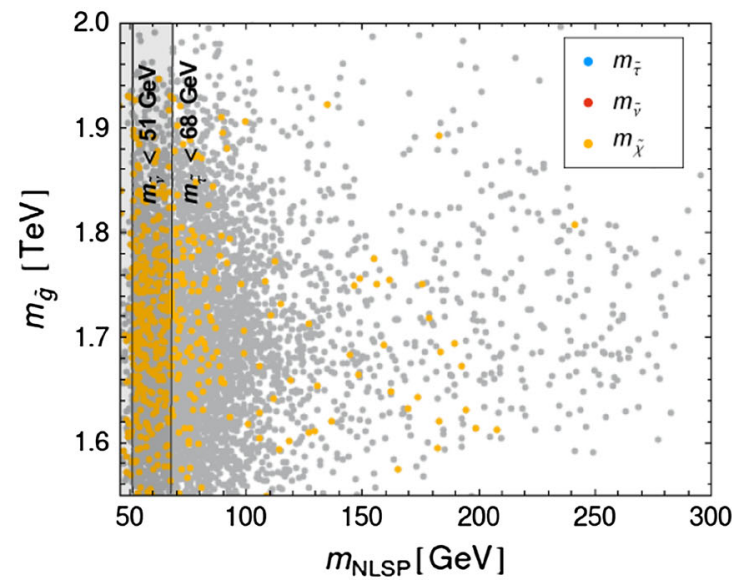

Fig. 7 The gluino mass $\left(m_{\tilde{g}}\right)$ as a function of NLSP mass $\left(m_{\mathrm{NLSP}}\right)$ for MI (left) and MII (right), where the colours indicate the type of NLSP as shown in the legend. The LEP exclusion limit for the case of the $\tilde{\tau}$

discussed previously, the quiver structure means that at the high scale the masses of uncoloured scalar particles lying on site A are suppressed, which can in particular result in light higgsinos or sleptons compared to minimal GMSB.

The phenomenology of the model depends decisively on the nature of the NLSP, as this decides which SM particle is present in the final state along with the gravitino $\tilde{G}$. ATLAS and CMS have recently made much progress on constraining gauge-mediated models, where they study final states containing missing transverse energy $\left(E_{T}^{\text {miss }}\right)$ due to the gravitino $(\tilde{G})$ escaping the detector. Bino-like NLSPs decay via $\tilde{\chi_{1}} \rightarrow \tilde{G} \gamma$, such that the signature is $\gamma \gamma+E_{T}^{\text {miss }}$, along with additional jets depending on whether the production process is $\tilde{g} \tilde{g}$ or $\tilde{\chi}_{1}^{0} \tilde{\chi}_{1}^{0}$ [83]. When higgsino-like, the NLSP instead decays to a Higgs which can be detected via $b$ jets, and a mixed higgsino-bino NLSP can be searched for via a

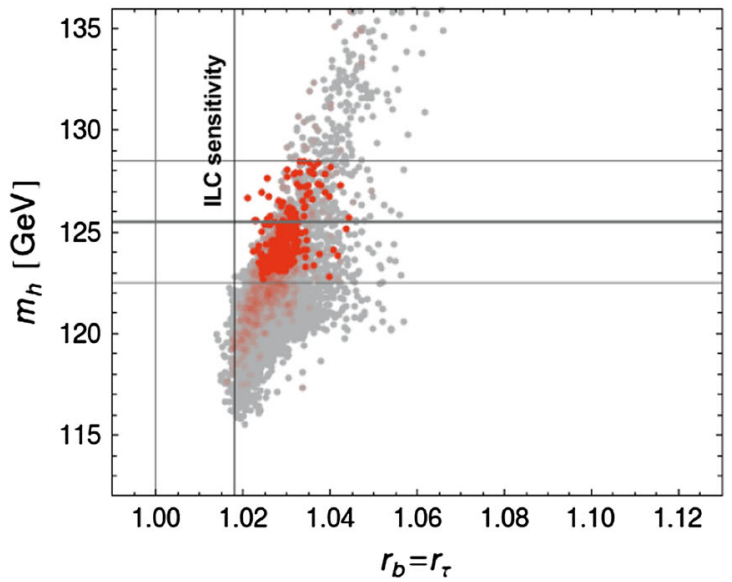

Grey points are excluded. The thick (thin) grey horizontal lines denote the central value (uncertainty) on $m_{h}$ and the vertical lines show the SM value $r_{b}=1$ and the projected ILC uncertainty of $2 \%$ (see text)

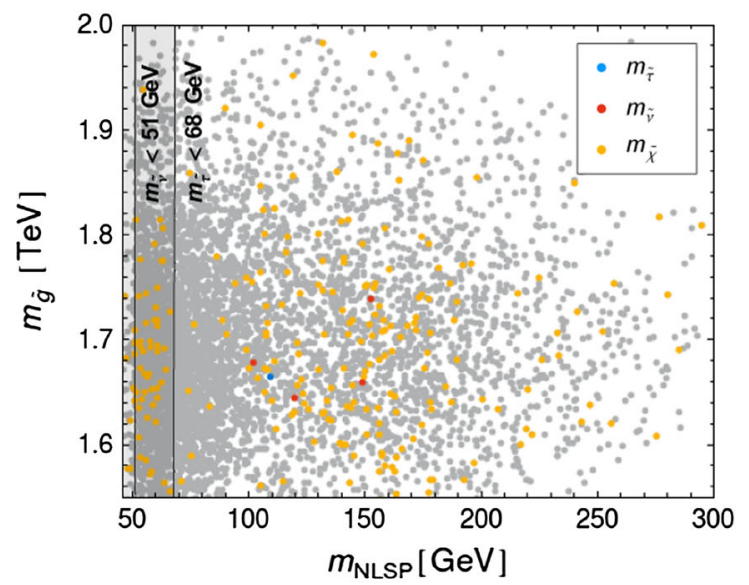

and $\tilde{v}$ NLSP is clearly marked, whereas the limit for $m_{\tilde{\chi}_{1}^{0}}$ is given by the $y$ axis. The grey points are excluded by experimental constraints as described in the text

$\gamma b \bar{b}+E_{T}^{\text {miss }}$ signature [84]. For stau or sneutrino NLSPs the $\tau$ or $v$ must be searched for in the final state.

In order to determine which experimental searches are relevant for these models, in Fig. 7 we examine the region of the $m_{\tilde{g}}-m_{\text {NLSP }}$ plane accessed by our scans, indicating the type of NLSP for each point, which we find may be the neutralino, stau or sneutrino. The LEP exclusion limits (see Sect. 4.2) for both the $\tilde{\tau}$ and $\tilde{v}$ NLSP are clearly marked, whereas the limit for $m_{\tilde{\chi}_{1}^{0}}$ is given by the y-axis. We find that for MII there are allowed points for which either the sneutrino or stau are the NLSP, however, for MI no such points were found. In MI, the generations of sfermions are treated equally, such that the staus lie close to the other sleptons. On the other hand in the case of MII, as mentioned earlier, above symmetry breaking the third generation sfermions are on site $\mathrm{A}$, whereas the lower generation ones on site B. This has the result that, 

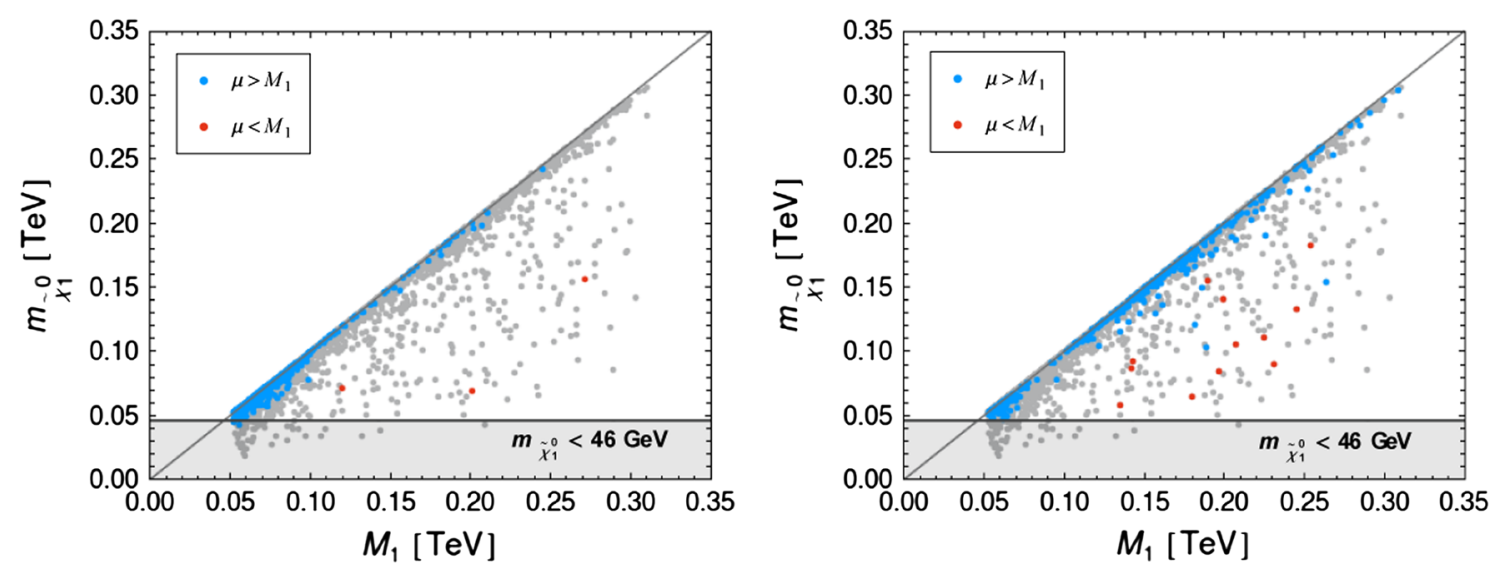

Fig. 8 The lightest neutralino mass $\left(m_{\tilde{\chi}_{1}^{0}}\right)$ as a function of $M_{1}$ for MI (left $)$ and MII (right). The grey points are excluded by experimental constraints. The grey diagonal line indicates $m_{\tilde{\chi}_{1}^{0}}=M_{1}$, and the horizontal line indicates the LEP exclusion limit as described in the text

as in the stop sector, the left-handed stau soft mass may be lower than the right-handed one, such that a sneutrino NLSP is possible. Therefore, although points in both models were found where the NLSP is the stau or sneutrino, only in MII do points survive the demanding constraints imposed on the Higgs mass and couplings due to measurements at the LHC, as illustrated in Fig. 8 .

As the lightest neutralino $\tilde{\chi}_{1}^{0}$ appears to be the favoured candidate for the NLSP, it is interesting to explore its composition as this will enlighten us as to which decay modes are preferred. Therefore in Fig. 8, we show the lightest neutralino mass, $m_{\tilde{\chi}_{1}^{0}}$, as a function of $M_{1}$. From this plot one can deduce whether $\tilde{\chi}_{1}^{0}$ is higgsino- or bino-like, respectively, depending on the higgsino and bino masses, approximately given by $\mu$ and $M_{1}$, respectively. The ubiquitous blue points indicate $\mu>M_{1}$ whereas the more rare red points, which are even absent for MI, show $\mu<M_{1}$. The grey points are excluded by Higgs and low-energy constraints, and the horizontal line demarcates the LEP-excluded region for $m_{\tilde{\chi}_{1}^{0}}$. In both MI and MII, the higgsino is rarely lighter than the bino, such that the NLSP is mostly bino-like or mixed bino-higgsino, while both can easily be below $300 \mathrm{GeV}$. This is interesting in light of the fact that experiments are sensitive to the nature of the neutralino NLSP, and the searches would therefore involve photons and/or Higgs bosons and missing transverse energy. Note that the feature of $\mu$ being light results in the model being less fine-tuned.

The experimental search strategy is not only dependent on the SM particle in the final state, but also on the decay length of the NLSP, $c \tau$. This can be approximated by [85],

$c \tau \sim \frac{16 \pi F^{2}}{m_{\mathrm{NLSP}}^{5}}$,

where we take $F=\Lambda M$. In the region of parameter space considered in this paper, the NLSP decays within the detec- tor. For the case of the neutralino NLSP decaying to a photon and gravitino, which is the prevalent case in both MI and MII, the excellent time measurement of the electromagnetic calorimeter in both ATLAS and CMS means that the time of arrival of the photon can be measured. If the NLSP decays immediately, i.e. if $c \tau<10^{-4} \mathrm{~m}$, then the decay is characterised as prompt, but otherwise it is non-prompt and it may be possible to deduce its decay length $[86,87]$. We therefore show the decay length of the NLSP in Fig. 9, as a function of the mass $m_{\mathrm{NLSP}}$.

From this figure we can confirm that the lightest neutralino NLSP may undergo both promp or non-prompt decays to the photon and the gravitino, although in MI fewer points survive for which the neutralino decays promptly.

The most important channels for these models at the LHC are therefore searches for photons and missing transverse energy, where the photons may be prompt or non-prompt. Here the dominant production would be electroweak, as our choice of $\Lambda_{3}$ is such that the gluon and squark pair production is suppressed. Studies so far by CMS have concentrated on strong production of the bino-like NLSP $[87,88]$, whereas ATLAS has considered the diphoton and missing transverse energy final state from direct electroweakino production, for the case of both promptly decaying [83] and long-lived neutralinos [86]. However, the bounds obtained by ATLAS are not directly applicable here, as they are presented for a specific point SPS8 [89], where the neutralino NLSP decays predominantly to the photon and gravitino which is not necessarily the case in our model, especially due to the fact that the higgsino is often light. Therefore the bounds on final states including Higgs bosons, studied in Refs. [90,91], must be taken into account. In order to constrain MII, one must further consider the stau and sneutrino NLSP, therefore final states involving $\tau \mathrm{s}$ and missing transverse energy are of interest [91,92]. It would be of great interest to combine all these excluded cross sections to extract precise exclusion bounds 


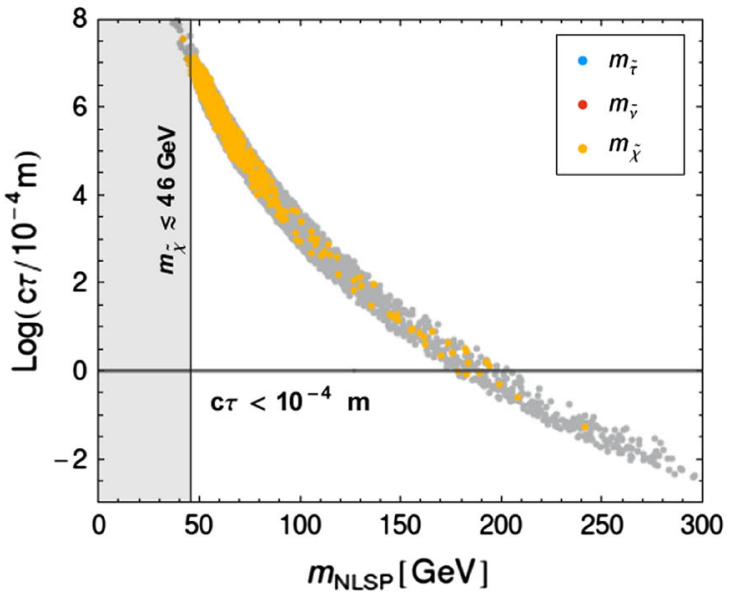

Fig. 9 The decay length $(c \tau)$ of the NLSP as a function of its mass $\left(m_{\mathrm{NLSP}}\right)$ for MI (left) and MII (right). Points for which $m_{\tilde{t}_{1}}<2 \mathrm{TeV}$ and which satisfy all constraints imposed are shown in yellow, whereas the

(along the lines of e.g. Ref. [93]), but this is beyond the scope of this paper. It nonetheless seems that for gauge-mediated models an interesting region of parameter space is starting to be probed, and we eagerly await further results.

\section{Conclusions and discussion}

In this paper we have examined phenomenological aspects of a minimal gauge extension of the MSSM containing two copies of the electroweak gauge group. Using state-of-the-art publicly available HEP tools, we have computed the two-loop RG equations for all parameters of two variants MI and MII of this basic setup, characterised by different assignments for the representation of the MSSM chiral superfields. Although the model may be amenable to any set of soft term boundary conditions, we have chosen to work within the framework of gauge-mediated supersymmetry breaking and we performed the RG evolution from the messenger scale down to the electroweak scale in order to compute the sparticle spectrum. We calculated the corresponding sparticle masses at one loop, the predicted Higgs boson mass at two loops and further investigated the predictions of the models MI and MII for the most relevant experimental observables.

As the extended gauge structure results in non-decoupled D-terms which increase the tree-level Higgs mass, the resulting spectrum can be more natural than in minimal GMSB. We further found that in order to be in agreement with Higgs mass constraints, while keeping the stop masses below $2 \mathrm{TeV}$, one must generate sufficiently large $\Delta$ (3.23). This requires the linking soft mass $m_{L}$ to be $\mathcal{O}(3-10)$ times higher than expected from the exact GMSB boundary conditions, which indicates a useful direction in which to extend this work on a theoretical level.

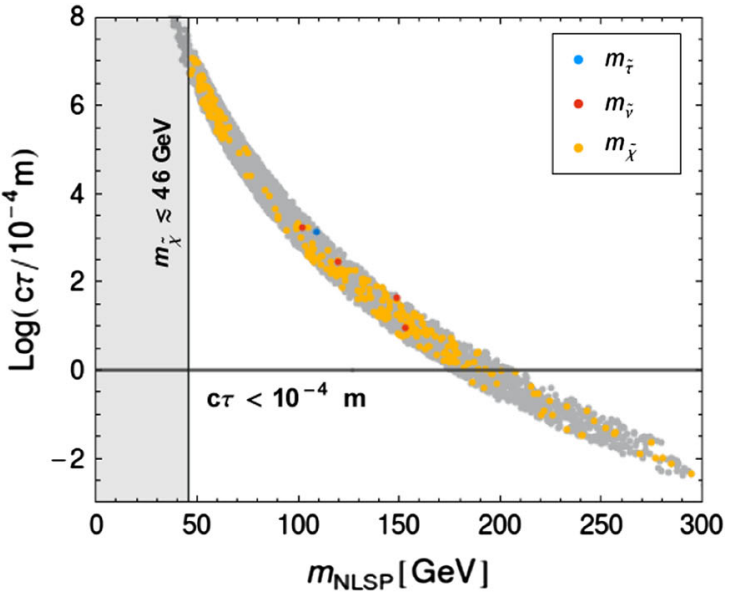

remaining excluded points are shown in grey. The vertical lines indicate the exclusion limits for staus and sneutrinos from LEP

We also found that both variants MI and MII of the model would have interesting phenomenological consequences at colliders, since they could be probed either indirectly through Higgs couplings measurements or via direct sparticle production. The Higgs couplings to down-type fermions deviate from the SM due to the $\Delta$ by $r_{b} \lesssim 6 \%$ for MI and $r_{b} \lesssim 4 \%$ for MII, and although at present this is well within experimental limits, such deviations should be measurable at a linear collider. As we have focussed on the region of parameter space where the coloured sector is $\sim 2 \mathrm{TeV}$ in order to evade bounds on squark and gluino production from the LHC, while the electroweak sector is kept below $1 \mathrm{TeV}$, the most promising production channel at the $\mathrm{LHC}$ is the direct production of electroweakinos. As the predominant NLSP is the bino-like neutralino, diphoton and missing transverse energy searches offer the most promising search perspectives, though for MII, the NLSP is not limited to the bino such that finals states containing $\tau$ or $h$ and missing transverse energy are also relevant. As the LHC exclusions are presented in terms of specific models, we are therefore keen to reinterpret these in order to understand how these bounds translate in the case of our model.

There are a number of ways in which this work may be extended. A first step, as mentioned earlier, would be to determine whether larger $\Delta \mathrm{s}$ can be realised without making $m_{L}$ a free parameter by including $\mathrm{U}(1)$ kinetic mixing or, more ambitiously, an additional SU(3). This could be achieved by means of the tools we have developed with the help of the publicly available package SARAH. It would further be of interest to study related models of flavour, or models with chiral non-decoupled D-terms at the same level of precision. By moving Higgs fields or generations onto different quiver sites, such models are relatively straightforward to implement in our setup. It would also be ideal to construct single regime 
models, in particular for cases where the phenomenology of additional light states may become relevant. Furthermore, not only are there $S O(N)$ and $S p(N)$ gauge extensions, but even more general quiver constructions, such as those with three or more sites [12,94], may also be implemented in full. The study of these models and their GUT completion is also a noble task from the perspective of string phenomenology which has so far been rather neglected.

Acknowledgments We would all like to thank Florian Staub for numerous communications on SARAH and Werner Porod on SPheno. M.M. would like to thank Martin Winkler, Jan Hajer, Kazuki Sakurai, Lisa Zeune, Florian Domingo, Kai Schmidt-Hoberg, Felix Brümmer and Jörg Jäckel for very useful discussions. A.G. would like to acknowledge enlightening discussions with Fawzi Boudjema, Geneviève Belanger, Björn Herrmann, Antonio Mariano, Kirtimaan Mohan, Pierre Salati and Paul Sorba. Our model package is available on request. M.M. is funded by the Alexander von Humboldt Foundation.

Open Access This article is distributed under the terms of the Creative Commons Attribution License which permits any use, distribution, and reproduction in any medium, provided the original author(s) and the source are credited.

Funded by $\mathrm{SCOAP}^{3}$ / License Version CC BY 4.0.

\section{Appendix A: Some further comments on the implementation}

In this section we include some useful comments on the implementation of the model.

\section{A.1: Fermion mixing and soft term matching}

In regime 1 there are many fermions that may mix together. The uncharged fermions are the diagonal linking fermions, the light and massive bino-type fermions, the uncharged light and massive wino-type fermions and finally the diagonal fermion of the $A$ adjoint superfield as well as the $K$ singlet fermion:

$$
\left(\chi_{L}^{1}, \chi_{\tilde{L}}^{1}, \chi_{L}^{4}, \chi_{\tilde{L}}^{4}, \tilde{B}_{L}, \tilde{B}_{M}, \tilde{W}_{L}^{3}, \tilde{W}_{M}^{3}, \psi_{A}^{1}, \psi_{K}\right)
$$

The lightest two of these states become the MSSM bino and uncharged wino. The mass matrices may be found in the of the model file in SARAH.

The charged fermions that mix together are the offdiagonal linking fermions, the charged wino-type light and massive gauginos and the off-diagonal $A$ superfield fermions:

$$
\left(\chi_{L}^{2}, \chi_{\tilde{L}}^{2}, \chi_{L}^{3}, \chi_{\tilde{L}}^{3}, \tilde{W}_{L}^{1}, \tilde{W}_{M}^{1}, \tilde{W}_{L}^{2}, \tilde{W}_{M}^{2}, \psi_{A}^{2}, \psi_{A}^{3}\right)
$$

the lightest two of these become the MSSM charged winos. The rest of the states, both scalar and fermion, of the linking fields $K, A, L, \tilde{L}$ are integrated out at the threshold scale between the first and second regime.

\section{A.2: MSSM Higgs and sfermion mixing matrices}

The non-decoupled D-terms of Sect. 3.4 appear in the tadpole equations as well as the Higgs mixing matrices. For the real components $\left(\phi_{d}, \phi_{u}\right),\left(\phi_{d}, \phi_{u}\right)$ we get

$m_{h}^{2}=\left(\begin{array}{cc}m_{11} & -\frac{1}{4} g_{12}^{2} v_{d} v_{u}-\operatorname{Re}\left[B_{\mu}\right] \\ -\frac{1}{4} g_{12}^{2} v_{d} v_{u}-\operatorname{Re}\left[B_{\mu}\right] & m_{22}\end{array}\right)$

where $g_{1}=g^{\prime}$ and for convenience we use $g_{12}^{2}=\left(g_{1}^{2}+g_{2}^{2}+\right.$ $\left.g_{1}^{2} \Delta_{1}^{2}+g_{2}^{2} \Delta_{2}^{2}\right)$.

$m_{11}=\frac{1}{8}\left(8 m_{H_{d}}^{2}+8|\mu|^{2}+g_{12}^{2}\left(3 v_{d}^{2}-v_{u}^{2}\right)\right)$

$m_{22}=\frac{1}{8}\left(8 m_{H_{u}}^{2}+8|\mu|^{2}-g_{12}^{2}\left(-3 v_{u}^{2}+v_{d}^{2}\right)\right)$

while for pseudo-scalar Higgses $\left(\sigma_{d}, \sigma_{u}\right),\left(\sigma_{d}, \sigma_{u}\right)$ the relevant expressions are

$m_{A^{0}}^{2}=\left(\begin{array}{cc}m_{11} & \operatorname{Re}\left[B_{\mu}\right] \\ \operatorname{Re}\left[B_{\mu}\right] & m_{22}\end{array}\right)+\xi_{Z} m_{Z}^{2}$

$m_{11}=\frac{1}{8}\left(8 m_{H_{d}}^{2}+8|\mu|^{2}+g_{12}^{2}\left(-v_{u}^{2}+v_{d}^{2}\right)\right)$

$m_{22}=\frac{1}{8}\left(8 m_{H_{u}}^{2}+8|\mu|^{2}-g_{12}^{2}\left(-v_{u}^{2}+v_{d}^{2}\right)\right)$

The mass matrix for the charged Higgses $\left(H_{d}^{-}, H_{u}^{+, *}\right)$, $\left(H_{d}^{-, *}, H_{u}^{+}\right)$reads

$m_{H^{-}}^{2}=\left(\begin{array}{cc}m_{11} & 1 \\ \frac{1}{4}\left(4 B_{\mu}+\left(g_{2}^{2}+g_{2}^{2} \Delta_{2}^{2}\right) v_{d} v_{u}\right) & \frac{1}{4}\left(4 B_{\mu}^{*}+\left(g_{2}^{2}+g_{2}^{2} \Delta_{2}^{2}\right) v_{d} v_{u}\right) \\ m_{22}\end{array}\right)$

$+\xi_{W^{-}} m_{W^{-}}^{2}$

$m_{11}=\frac{1}{8}\left(8 m_{H_{d}}^{2}+8|\mu|^{2}+g_{12}^{2} v_{d}^{2}+\hat{g}_{12}^{2} v_{u}^{2}\right)$

$m_{22}=\frac{1}{8}\left(8 m_{H_{u}}^{2}+8|\mu|^{2}+g_{12}^{2} v_{u}^{2}+\hat{g}_{12}^{2} v_{d}^{2}\right)$

where we have used the abbreviation $\hat{g}_{12}^{2}=\left(-g_{1}^{2}-g_{1}^{2} \Delta_{1}^{2}+\right.$ $g_{2}^{2}+g_{2}^{2} \Delta_{2}^{2}$ ), and in all the above expressions, the $\xi$-terms are gauge-dependent contributions (and we work in Feynman gauge throughout this paper).

For completeness, we also include the mixing matrix $M_{\tilde{f}}$ of a generic sfermion $\tilde{f}$ which may be a squark or charged slepton. This matrix takes the form

$M_{\tilde{f}}=\left(\begin{array}{cc}m_{\tilde{f}_{L}}^{2}+m_{f}^{2}+\hat{M}_{Z}^{2}\left(I_{3}^{f}-Q_{f} s_{W}^{2}\right) & m_{f} X_{f}^{*} \\ m_{f} X_{f} & m_{\tilde{f}_{R}}^{2}+m_{f}^{2}+\hat{M}_{Z}^{2} Q_{f} s_{W}^{2}\end{array}\right)$,

for $s_{w}=\sin \theta_{W}$ where $\theta_{W}$ is the Weinberg weak mixing angle, and we make use of the abbreviation $\hat{M}_{Z}^{2} \equiv$ $m_{Z}^{2} \cos 2 \beta$. The off-diagonal element $X_{f}$ is defined in terms of the trilinear coupling $A_{f}$ via

$X_{f}=A_{f}-\mu^{*}\{\cot \beta, \tan \beta\}$, 
where $\cot \beta$ applies for the up-type quarks, $f=u, c, t$, and $\tan \beta$ applies for the down-type fermions, $f=d, s, b, e, \mu, \tau$. Note that $m_{f}, Q_{f}$ and $I_{3}^{f}$ are the mass, charge and isospin projection of the fermion $f$, respectively. On diagonalisation of this matrix one obtains the light and heavy sfermion masses $m_{\tilde{f}_{1}}$ and $m_{\tilde{f}_{2}}$.

\section{A.3: Renormalisation group equations}

We evolved the model down from the messenger scale $M$, to a threshold scale $T_{\text {scale, }}$, which is associated with the masses of the linking field states $O\left(m_{v}^{2}\right)$. The two-loop renormalisation group equations were used in both regimes 1 and 2 , along with one-loop finite energy corrections and two-loop anomalous dimensions. The beta functions of the gauge couplings of the first regime at one loop are

$\beta_{g_{a}} \equiv \frac{\mathrm{d}}{\mathrm{d} t} g_{a}=\frac{b_{a}}{16 \pi^{2}} g_{a}^{3}$ with $b_{a}=\left(\frac{39}{5}, \frac{6}{5},-2,3,-3\right)$

where $a=\mathrm{U}(1)_{A}, \mathrm{U}(1)_{B}, \mathrm{SU}(2)_{A}, \mathrm{SU}(2)_{B}, \mathrm{SU}(3)$, which may be compared with the MSSM regime where

$b_{a}=(33 / 5,1,-3)$.

Let us also track the top Yukawas using the "only third family approximation",

$$
\begin{aligned}
\beta_{y_{t}}^{1} & \equiv \frac{\mathrm{d}}{\mathrm{d} t} y_{t} \\
\simeq & \frac{y_{t}}{16 \pi^{2}}\left[4 y_{t}^{*} y_{t}+y_{b}^{*} y_{b}-\frac{16}{3} g_{3}^{2}-3 g_{A 2}^{2}-\frac{13}{15} g_{A 1}^{2}\right] .
\end{aligned}
$$

In the second regime these become

$$
\begin{aligned}
\beta_{y_{t}} & \equiv \frac{\mathrm{d}}{\mathrm{d} t} y_{t} \\
\simeq & \frac{y_{t}}{16 \pi^{2}}\left[6 y_{t}^{*} y_{t}+y_{b}^{*} y_{b}-\frac{80}{15} g_{3}^{2}-3 g_{2}^{2}-\frac{13}{15} g_{1}^{2}\right] .
\end{aligned}
$$

In the first regime we find the trilinear $A_{t}$ coupling to be

$$
\begin{gathered}
16 \pi^{2} \frac{\mathrm{d}}{\mathrm{d} t} A_{t} \simeq A_{t}\left[9 y_{t}^{*} y_{t}+y_{b}^{*} y_{b}-\frac{16}{3} g_{3}^{2}-3 g_{A 2}^{2}-\frac{13}{15} g_{A 1}^{2}\right] \\
+y_{t}\left[\frac{32}{3} g_{3}^{2} m_{\tilde{g}}+6 g_{A 2}^{2} m_{\tilde{W}_{A}}+\frac{26}{15} g_{A 1}^{2} m_{\tilde{B}_{A}}\right]+2 a_{b} y_{b}^{*} y_{t}
\end{gathered}
$$

whereas in the MSSM

$$
\begin{gathered}
16 \pi^{2} \frac{\mathrm{d}}{\mathrm{d} t} A_{t} \simeq A_{t}\left[18 y_{t}^{*} y_{t}+y_{b}^{*} y_{b}-\frac{16}{3} g_{3}^{2}-3 g_{2}^{2}-\frac{13}{15} g_{1}^{2}\right] \\
+y_{t}\left[\frac{32}{3} g_{3}^{2} m_{\tilde{g}}+6 g_{2}^{2} m_{\tilde{W}}+\frac{26}{15} g_{1}^{2} m_{\tilde{B}}\right]+2 a_{b} y_{b}^{*} y_{t} .
\end{gathered}
$$

Let us also look at how the gauginos obtain soft mases. The one-loop beta functions for the B-site gaugino soft masses are given by

$\beta_{m_{\tilde{B}_{B}}}^{1}=\frac{12}{5} g_{B 1}^{2} m_{\tilde{B}_{B}} \beta_{m_{\tilde{W}_{B}}}^{1}=-4 g_{B 2}^{2} m_{\tilde{W}_{B}} \beta_{m_{\tilde{g}}}^{1}=-6 g_{3}^{2} m_{\tilde{g}}$.

For the A-site gauginos they are given by

$\beta_{m_{\tilde{B}_{A}}}^{1}=\frac{78}{5} g_{A 1}^{2} m_{\tilde{B}_{A}} \beta_{m_{\tilde{W}_{A}}}^{1}=6 g_{A 2}^{2} m_{\tilde{W}_{A}}$.

Even though the A-site gaugino masses are vanishing at the messenger scale $M$ the two-loop contributions which typically depend on all the other gaugino soft mases, feedback into the one-loop contributions. Finally the supersymmetric Dirac masses associated with the quiver structure will lift their mass eigenstates. The two-loop equations are given by

$$
\begin{aligned}
\beta_{m_{\tilde{B}_{B}}}^{(2)}= & \frac{6}{25} g_{B 1}^{2}\left(12 g_{B 1}^{2} m_{\tilde{B}_{B}}+30 g_{2 A}^{2}\left(m_{\tilde{W}_{A}}+m_{\tilde{W}_{B}}\right)\right. \\
& +30 g_{B 2}^{2}\left(m_{\tilde{B}_{B}}+m_{\tilde{W}_{B}}\right)+6 g_{A 1}^{2}\left(m_{\tilde{B}_{A}}+m_{\tilde{B}_{B}}\right) \\
& \left.-30 Y_{A}^{*}\left(m_{\tilde{B}_{B}} Y_{A}-T_{A}\right)+5 Y_{K}^{*}\left(T_{K}-m_{\tilde{B}_{B}} Y_{K}\right)\right)
\end{aligned}
$$$$
\beta_{m_{\tilde{B}_{A}}^{(2)}}^{(2)}=\frac{4}{75} g_{A 1}^{2}\left(-\frac{270}{2} Y_{A}^{*}\left(m_{\tilde{B}_{A}} Y_{A}-T_{A}\right)\right.
$$$$
-\frac{45}{2} Y_{K}^{*}\left(m_{\tilde{B}_{A}} Y_{K}-T_{K}\right)+620 g_{3}^{2} m_{\tilde{B}_{A}}
$$$$
+650 g_{A 1}^{2} m_{\tilde{B}_{A}}+315 g_{A 2}^{2} m_{\tilde{B}_{A}}+27 g_{B 1}^{2} m_{\tilde{B}_{A}}
$$$$
+135 g_{B 2}^{2} m_{\tilde{B}_{A}}+315 g_{A 2}^{2} m_{\tilde{W}_{A}}
$$$$
+27 g_{B 1}^{2} m_{\tilde{B}_{B}}+135 g_{B 2}^{2} m_{\tilde{W}_{B}}+620 g_{3}^{2} m_{\tilde{g}}
$$$$
-35 m_{\tilde{B}_{A}} \operatorname{Tr}\left(Y_{d} Y_{d}^{\dagger}\right)-135 m_{\tilde{B}_{A}} \operatorname{Tr}\left(Y_{e} Y_{e}^{\dagger}\right)
$$$$
-65 m_{\tilde{B}_{A}} \operatorname{Tr}\left(Y_{u} Y_{u}^{\dagger}\right)+35 \operatorname{Tr}\left(Y_{d}^{\dagger} T_{d}\right)
$$$$
\left.+135 \operatorname{Tr}\left(Y_{e}^{\dagger} T_{e}\right)+65 \operatorname{Tr}\left(Y_{u}^{\dagger} T_{u}\right)\right)
$$

$\beta_{m_{\tilde{W}_{A}}^{(2)}}^{(2)} \frac{2}{5} g_{A 2}^{2}\left(15 g_{A 1}^{2} m_{\tilde{B}_{A}}+120 g_{3}^{2} m_{\tilde{W}_{A}}+15 g_{A 1}^{2} m_{\tilde{W}_{A}}\right.$ $+390 g_{A 2}^{2} m_{\tilde{W}_{A}}+6 g_{B 1}^{2} m_{\tilde{W}_{A}}+30 g_{B 2}^{2} m_{\tilde{W}_{A}}$

$+6 g_{B 1}^{2} m_{\tilde{B}_{B}}+30 g_{B 2}^{2} m_{\tilde{W}_{B}}+120 g_{3}^{2} m_{\tilde{g}}$

$-30 Y_{A}^{*}\left(m_{\tilde{W}_{A}} Y_{A}-T_{A}\right)+Y_{K}^{*}\left(-5 m_{\tilde{W}_{A}} Y_{K}+5 T_{K}\right)$

$-10 m_{\tilde{W}_{A}} \operatorname{Tr}\left(Y_{d} Y_{d}^{\dagger}\right)-10 m_{\tilde{W}_{A}} \operatorname{Tr}\left(Y_{e} Y_{e}^{\dagger}\right)$

$-10 m_{\tilde{W}_{A}} \operatorname{Tr}\left(Y_{u} Y_{u}^{\dagger}\right)+10 \operatorname{Tr}\left(Y_{d}^{\dagger} T_{d}\right)$

$\left.+10 \operatorname{Tr}\left(Y_{e}^{\dagger} T_{e}\right)+10 \operatorname{Tr}\left(Y_{u}^{\dagger} T_{u}\right)\right)$

$$
\begin{aligned}
\beta_{m_{\tilde{W}_{B}}^{(2)}}^{(2)} & \frac{2}{5} g_{B 2}^{2}\left(6 g_{A 1}^{2} m_{\tilde{B}_{A}}+30 g_{A 2}^{2} m_{\tilde{W}_{A}}+6 g_{B 1}^{2} m_{\tilde{B}_{B}}\right. \\
& +6 g_{A 1}^{2} m_{\tilde{W}_{B}}+30 g_{A 2}^{2} m_{\tilde{W}_{B}} \\
& +6 g_{B 1}^{2} m_{\tilde{W}_{B}}+140 g_{B 2}^{2} m_{\tilde{W}_{B}}-70 Y_{A}^{*}\left(m_{\tilde{W}_{B}} Y_{A}-T_{A}\right)
\end{aligned}
$$




$$
\begin{aligned}
& \left.+Y_{K}^{*}\left(-5 m_{\tilde{W}_{B}} Y_{K}+5 T_{K}\right)\right) \\
\beta_{m_{\tilde{g}}}^{(2)}= & \frac{2}{15} g_{3}^{2}\left(33 g_{A 1}^{2} m_{\tilde{B}_{A}}+135 g_{A 2}^{2} m_{\tilde{W}_{A}}+420 g_{3}^{2} m_{\tilde{g}}\right. \\
& +33 g_{A 1}^{2} m_{\tilde{g}}+135 g_{A 2}^{2} m_{\tilde{g}}-20 m_{\tilde{g}} \operatorname{Tr}\left(Y_{d} Y_{d}^{\dagger}\right) \\
& \left.-20 m_{\tilde{g}} \operatorname{Tr}\left(Y_{u} Y_{u}^{\dagger}\right)+20 \operatorname{Tr}\left(Y_{d}^{\dagger} T_{d}\right)+20 \operatorname{Tr}\left(Y_{u}^{\dagger} T_{u}\right)\right) .
\end{aligned}
$$

The soft masses for the quiver module run too:

$$
\begin{aligned}
\beta_{m_{A}^{2}}^{(1)}= & -8 g_{B 2}^{2}\left|m_{\tilde{W}_{B}}\right|^{2}+4\left(m_{A}^{2}+m_{L}^{2}+m_{\tilde{L}}^{2}\right)\left|Y_{A}\right|^{2}+4\left|T_{A}\right|^{2} \\
\beta_{m_{K}^{2}}^{(1)}= & +2\left(m_{K}^{2}+m_{L}^{2}+m_{\tilde{L}}^{2}\right)\left|Y_{K}\right|^{2}+2\left|T_{K}\right|^{2} \\
\beta_{m_{\tilde{L}}^{2}}^{(1)} & =\frac{1}{10}\left(-12 g_{A 1}^{2}\left|m_{\tilde{B}_{A}}\right|^{2}-60 g_{A 2}^{2}\left|m_{\tilde{W}_{A}}\right|^{2}-12 g_{B 1}^{2}\left|m_{\tilde{B}_{B}}\right|^{2}\right. \\
& -60 g_{B 2}^{2}\left|m_{\tilde{W}_{B}}\right|^{2}+30\left(m_{A}^{2}+m_{L}^{2}+m_{\tilde{L}}^{2}\right)\left|Y_{A}\right|^{2} \\
& +5\left(m_{K}^{2}+m_{L}^{2}+m_{\tilde{L}}^{2}\right)\left|Y_{K}\right|^{2} \\
& \left.+30\left|T_{A}\right|^{2}+5\left|T_{K}\right|^{2}+6 g_{A 1}^{2} \sigma_{1,3}\right),
\end{aligned}
$$

where by $\sigma_{1,3}$ we denote the soft mass combination

$$
\begin{aligned}
\sigma_{1,3}= & -2 \operatorname{Tr}\left(m_{u}^{2}\right)-2 m_{L}^{2}+2 m_{\tilde{L}}^{2}-m_{H_{d}}^{2}+m_{H_{u}}^{2}+\operatorname{Tr}\left(m_{d}^{2}\right) \\
& +\operatorname{Tr}\left(m_{e}^{2}\right)-\operatorname{Tr}\left(m_{l}^{2}\right)+\frac{1}{3} \operatorname{Tr}\left(m_{q}^{2}\right) .
\end{aligned}
$$

The VEV of the linking fields runs as well:

$$
\begin{aligned}
\beta_{v}^{(1)}= & \frac{v}{20}\left(-30\left|Y_{A}\right|^{2}-5\left|Y_{K}\right|^{2}+3 g_{A 1}^{2}\right. \\
& \left.+15 g_{A 2}^{2}+3 g_{B 1}^{2}+15 g_{B 2}^{2}\right)(1+\xi) .
\end{aligned}
$$

Further equations may be found in the pdf for this model, including all anomalous dimensions and beta functions. At the electroweak scale one finds

$$
\begin{aligned}
|\mu|^{2}= & \frac{1}{8\left(v_{d}^{2}-v_{u}^{2}\right)}\left(-8 m_{H_{d}}^{2} v_{d}^{2}+8 m_{H_{u}}^{2} v_{u}^{2}-g_{1}^{2} \Delta_{1}^{2} v_{d}^{4}\right. \\
& -g_{2}^{2} \Delta_{2}^{2} v_{d}^{4}+g_{1}^{2} \Delta_{1}^{2} v_{u}^{4}+g_{2}^{2} \Delta_{2}^{2} v_{u}^{4}+g_{2}^{2} v_{u}^{4} \\
& \left.-g_{2}^{2} v_{d}^{4}-g_{1}^{2} v_{d}^{4}+g_{1}^{2} v_{u}^{4}\right)
\end{aligned}
$$

and

$$
\begin{aligned}
B_{\mu}=- & \frac{v_{d} v_{u}}{4\left(v_{d}^{2}-v_{u}^{2}\right)}\left(4 m_{H_{d}}^{2}-4 m_{H_{u}}^{2}\right. \\
& \left.+\left(g_{1}^{2}+g_{2}^{2}+g_{1}^{2} \Delta_{1}^{2}+g_{2}^{2} \Delta_{2}^{2}\right)\left(v_{d}^{2}-v_{u}^{2}\right)\right) .
\end{aligned}
$$

which are after all used to minimise the electroweak tadpole equations.

\section{A.4: Threshold effects}

We integrate out various states at the threshold between the two regimes. These include the fermions discussed above as well as all linking scalars and scalars of $K$ and $A$. To implement this correctly, with two-loop RGEs, we edited by hand the SPheno code to properly account for the finite shifts and the mass orderings of the particles integrated out, given by

$g_{i} \rightarrow g_{i}\left[1 \pm \frac{g_{i}^{2} b_{\text {state }}^{i}(R)}{8 \pi^{2}} \ln \left(\frac{M_{\text {state }}}{M_{T}}\right)\right]$,

to account for our particular matter content and

$m_{\tilde{g}} \rightarrow m_{\tilde{g}}\left[1 \pm \frac{g_{i}^{2} b_{\text {state }}^{i}(R)}{8 \pi^{2}} \ln \left(\frac{M_{\text {state }}}{M_{T}}\right)\right]$

for the gluino shift between regimes. The other soft mass parameters for the bino and winos are matched as the lightest states as explained in Appendix A.1. The shifts for each field component are given by

$b_{\text {state }}(R)=\{11 / 3,-2 / 3,-1 / 3,-1 / 6\} \times \frac{T(R)}{D(R)}$.

The numbers are associated to a gauge boson, Weyl fermion, complex and real scalar, respectively. $T(R)$ is the index (half the Dynkin index $I(R))$, such that $T(\square)=1 / 2, T(\operatorname{Adj})=$ $N_{c}$ ). It is divided by the dimension of the representation $\mathrm{D}(\mathrm{R})$ as each shift is for the component of the field and not the full multiplet, in SARAH. The massive gauge fields are integrated out either on their own or by including them with the finite shifts of the real eaten Goldstone modes.

\section{A.5: Soft mass function}

In the SARAH package we included, in the form of a Fortran function, the generalisation of the usual mGMSB formula $f(x)$ to the case of a two-site quiver model: $s(x, y)$. The analytic expression is given by

$$
\begin{aligned}
s(x, y)= & \frac{1}{2 x^{2}}\left(s_{0}+\frac{s_{1}+s_{2}}{y^{2}}+s_{3}+s_{4}+s_{5}\right) \\
& +(x \rightarrow-x),
\end{aligned}
$$

where

$$
\begin{aligned}
s_{0}= & 2(1+x)\left(\log (1+x)-2 \operatorname{Li}_{2}\left(\frac{x}{1+x}\right)\right. \\
& \left.+\frac{1}{2} \operatorname{Li}_{2}\left(\frac{2 x}{1+x}\right)\right), \\
s_{1}= & -4 x^{2}-2 x(1+x) \log ^{2}(1+x)-x^{2} \operatorname{Li}_{2}\left(x^{2}\right), \\
s_{2}= & 8(1+x)^{2} h\left(\frac{y^{2}}{1+x}, 1\right)
\end{aligned}
$$




$$
\begin{aligned}
& -4 x(1+x) h\left(\frac{y^{2}}{1+x}, \frac{1}{1+x}\right) \\
& -4 x h\left(y^{2}, 1+x\right)-8 h\left(y^{2}, 1\right), \\
s_{3}= & -2 h\left(\frac{1}{y^{2}}, \frac{1}{y^{2}}\right)-2 x h\left(\frac{1+x}{y^{2}}, \frac{1}{y^{2}}\right) \\
& +2(1+x) h\left(\frac{1+x}{y^{2}}, \frac{1+x}{y^{2}}\right), \\
s_{4}= & (1+x)\left(2 h\left(\frac{y^{2}}{1+x}, \frac{1}{1+x}\right)-h\left(\frac{y^{2}}{1+x}, 1\right)\right. \\
& \left.-h\left(\frac{y^{2}}{1+x}, \frac{1-x}{1+x}\right)\right), \\
s_{5}= & 2 h\left(y^{2}, 1+x\right)-2 h\left(y^{2}, 1\right) .
\end{aligned}
$$

The function $h$ is given by the integral

$h(a, b)=\int_{0}^{1} \mathrm{~d} x\left(1+\operatorname{Li}_{2}\left(1-\mu^{2}\right)-\frac{\mu^{2}}{1-\mu^{2}} \log \mu^{2}\right)$.

The dilogarithm is defined as $\operatorname{Li}_{2}(x)=-\int_{0}^{1} \frac{\mathrm{d} t}{t} \log (1-x t)$ with

$\mu^{2}=\frac{a x+b(1-x)}{x(1-x)}, \quad a=m_{1}^{2} / m_{0}^{2}, \quad b=m_{2}^{2} / m_{0}^{2}$.

So as not to introduce IR divergent pieces it is best to first evaluate terms with massless propagators. In that case the function $h$ simplifies to $h(0, b)=1+\operatorname{Li}_{2}(1-\mathrm{b})$ and has the symmetry $h(b, 0)=h(0, b)$. For four massive poles, the analytic expression for $h$ is used in SARAH,

$$
\begin{aligned}
& h(a, b)=1-\frac{\log a \log b}{2}-\frac{a+b-1}{\sqrt{\Delta}}\left(\operatorname{Li}_{2}\left(-\frac{u_{2}}{v_{1}}\right)\right. \\
& +\operatorname{Li}_{2}\left(-\frac{v_{2}}{u_{1}}\right)+\frac{1}{4} \log ^{2} \frac{u_{2}}{v_{1}}+\frac{1}{4} \log ^{2} \frac{v_{2}}{u_{1}}+\frac{1}{4} \log ^{2} \frac{u_{1}}{v_{1}} \\
& \left.\quad-\frac{1}{4} \log ^{2} \frac{u_{2}}{v_{2}}+\frac{\pi^{2}}{6}\right)
\end{aligned}
$$

where

$$
\begin{gathered}
\Delta=1-2(a+b)+(a-b)^{2}, \\
u_{1,2}=\frac{1+b-a \pm \sqrt{\Delta}}{2}, \\
v_{1,2}=\frac{1-b+a \pm \sqrt{\Delta}}{2} .
\end{gathered}
$$

For illustration, in Fig. 10 we depict $s(x, y)$ as a function of the parameter $x$ for some indicative, fixed values of $y$.

\section{A.6: Generalising non-decoupled D-terms}

Previously non-decoupled D-terms have been used to explore both vector-like and chiral D-terms for the MSSM Higgses

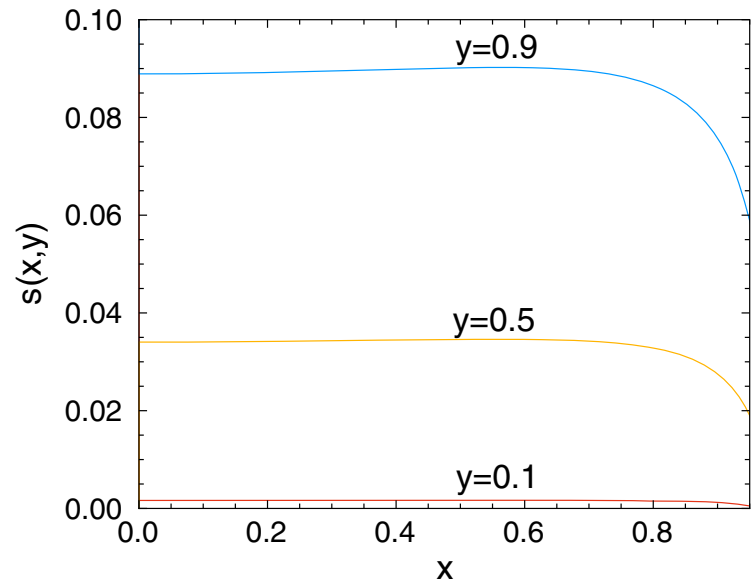

Fig. 10 We show $s(x, y)$ as a function of the parameter $x$ for fixed values of $y$ as indicated

$[3,59]$. It is actually the case that this effective action effects all fields charged under the relevant symmetries and as a result, there will be effective terms for squarks and sleptons too. This point has so far not been mentioned in the literature. We therefore supply a more general derivation for the twosite quiver, whose main result is (7.52), although we only include the Higgs contributions in our study. It may also be extend to the three-site case.

For two abelian or non-abelian gauge groups $G_{A} \times G_{B}$ that break to the diagonal, one may write canonical kinetic terms for chiral superfields charged under only site A or site B:

$\mathcal{L} \supset \int \mathrm{d}^{4} \theta\left(\sum_{i} A_{i}^{\dagger} e^{g_{a} V_{a}} A_{i}+\sum_{j} B_{j}^{\dagger} e^{g_{b} V_{b}} B_{j}\right)$.

After breaking to the diagonal, there is a massless and massive vector multiplet

$V_{G}=\frac{g_{a} V_{b}+g_{b} V_{a}}{\sqrt{g_{a}^{2}+g_{b}^{2}}} \quad V_{H}=\frac{-g_{a} V_{a}+g_{b} V_{b}}{\sqrt{g_{a}^{2}+g_{b}^{2}}}$

and usefully for computing the equation of motion, there is a mass term in the Kähler potential,

$\mathcal{L} \supset \int \mathrm{d}^{4} \theta m_{V}^{2} V_{H}^{2}+\cdots$

One may in fact add a number of soft mass terms

$$
\begin{aligned}
\mathcal{L} & \supset \int \mathrm{d}^{4} \theta\left(m_{\chi} m_{V}^{2} \theta^{2}+\bar{m}_{\chi} m_{V}^{2} \bar{\theta}^{2}-\frac{1}{2} m_{V}^{2} m_{s}^{2} \theta^{4}\right) V^{2} \\
& +\int \mathrm{d}^{2} \theta m_{\lambda} W_{\alpha}^{2}+\int \mathrm{d}^{2} \bar{\theta} \bar{m}_{\lambda} \bar{W}_{\dot{\alpha}}^{2}
\end{aligned}
$$

to parameterise the soft breaking fermion $\chi$, the real uneaten scalar, and the Majorana soft mass for $\lambda$, respectively. In terms of standard current multiplets satisfying $D^{2} \mathcal{J}=0$, the Kähler potential may be written to leading order in $V_{H}$ as 
$g_{d}\left(\frac{g_{a}}{g_{b}}\right) \mathcal{J}_{a} V_{H}+g_{d}\left(\frac{g_{b}}{g_{a}}\right) \mathcal{J}_{b} V_{H}+\cdots$,

where $\mathcal{J}_{a / b}$ are the currents that contain all the fields charged under site a or site $b$ :

$$
\begin{aligned}
\mathcal{J}^{A}= & J^{A}+i \theta j^{A}-i \bar{\theta} \bar{j}^{A}-\theta \sigma^{\mu} \bar{\theta} j_{\mu}^{A}+\frac{1}{2} \theta \theta \bar{\theta} \bar{\sigma}^{\mu} \partial_{\mu} j^{A} \\
& -\frac{1}{2} \bar{\theta} \bar{\theta} \theta \sigma^{\mu} \partial_{\mu} \bar{j}^{A}-\frac{1}{4} \theta \theta \bar{\theta} \bar{\theta} \square J^{A},
\end{aligned}
$$

with the leading term being the scalar current $J^{A}=$ $\sum_{i} \phi_{i}^{\dagger} T^{A} \phi_{i}$. The diagonal gauge group coupling is $g_{d}=$ $g_{\mathrm{SM}}$. The effective lagrangian after integrating out $V_{H}$ will then be of the form

$\mathcal{L}_{\text {eff }}=\int \mathrm{d}^{4} \theta\left(\sum_{i} A_{i}^{\dagger} e^{g_{d} V_{d}} A_{i}+\sum_{j} B_{j}^{\dagger} e^{g_{d} V_{d}} B_{j}\right)+\mathcal{O}$

where $\mathcal{O}$ is the effective super operator

$$
\begin{aligned}
\mathcal{O} & =g_{d}^{2} \int \mathrm{d}^{4} \theta\left(\frac{1}{m_{V}^{2}}-\frac{m_{s}^{2} \theta^{4}}{m_{V}^{2}+m_{s}^{2}}\right) \\
& \times \sum_{A}\left[\left(\frac{g_{a}}{g_{b}}\right) \mathcal{J}_{a}^{A}-\left(\frac{g_{b}}{g_{a}}\right) \mathcal{J}_{b}^{A}\right]^{2}
\end{aligned}
$$

with a sum over $A$ generators. It is this effective super operator (7.52) that is the most general expression for the nondecoupled D-term of the two-site quiver, and produces both the chiral and vector-like non-decoupled D-terms as limiting cases. Explicitly for model MI the scalar currents are given by

$$
\begin{aligned}
J_{\mathrm{U}(1)_{A}}= & \frac{1}{2} H_{u}^{\dagger} H_{u}-\frac{1}{2} H_{d}^{\dagger} H_{d}-\frac{1}{2} \tilde{l}^{\dagger} \tilde{l}+\frac{1}{6} \tilde{q}^{\dagger} \tilde{q} \\
& +\frac{1}{3} \tilde{d}^{\dagger} \tilde{d}-\frac{2}{3} \tilde{u}^{\dagger} \tilde{u}+\tilde{e}^{\dagger} \tilde{e}, \quad J_{\mathrm{U}(1)_{B}}=0 \\
J_{\mathrm{SU}(2)_{A}}^{A}= & \frac{1}{2}\left(H_{u}^{\dagger} \sigma^{A} H_{u}+H_{d}^{\dagger} \sigma^{A} H_{d}+\tilde{q}^{\dagger} \sigma^{A} \tilde{q}+\tilde{l}^{\dagger} \sigma^{A} \tilde{l}\right), \\
& J_{\mathrm{SU}(2)_{B}}^{A}=0
\end{aligned}
$$

with all flavour and colour indices implicitly traced. For MII one finds

$$
\begin{aligned}
& J_{\mathrm{U}(1)_{A}}=\frac{1}{2} H_{u}^{\dagger} H_{u}-\frac{1}{2} H_{d}^{\dagger} H_{d} \\
& +\left[-\frac{1}{2} \tilde{l}^{\dagger} \tilde{l}+\frac{1}{6} \tilde{q}^{\dagger} \tilde{q}+\frac{1}{3} \tilde{d}^{\dagger} \tilde{d}-\frac{2}{3} \tilde{u}^{\dagger} \tilde{u}+\tilde{e}^{\dagger} \tilde{e}\right]_{3} \\
& J_{\mathrm{U}(1)_{B}}=\left[-\frac{1}{2} \tilde{l}^{\dagger} \tilde{l}+\frac{1}{6} \tilde{q}^{\dagger} \tilde{q}+\frac{1}{3} \tilde{d}^{\dagger} \tilde{d}-\frac{2}{3} \tilde{u}^{\dagger} \tilde{u}+\tilde{e}^{\dagger} \tilde{e}\right]_{1,2} \\
& J_{\mathrm{SU}(2)_{A}}^{A}=\frac{1}{2}\left(H_{u}^{\dagger} \sigma^{A} H_{u}+H_{d}^{\dagger} \sigma^{A} H_{d}\right) \\
& +\frac{1}{2}\left[\tilde{q}^{\dagger} \sigma^{A} \tilde{q}+\tilde{l}^{\dagger} \sigma^{A} \tilde{l}\right]_{3}, \\
& J_{\mathrm{SU}(2)_{B}}^{A}=+\frac{1}{2}\left[\tilde{q}^{\dagger} \sigma^{A} \tilde{q}+\tilde{l}^{\dagger} \sigma^{A} \tilde{l}\right]_{1,2} .
\end{aligned}
$$

The effective action containing all fields charged under the gauge groups may be included in regime 2 of the SARAH model file and due to the square in (7.52) these terms generate both mass shifts for all charged squarks and sleptons as well as additional quartic vertices. These additional contributions to branching ratios would need to be included in a precision study involving Higgs and sfermion decays, as might be accessible to an $e^{+}, e^{-}$collider such as the ILC. This effect, albeit subtle, if measured precisely enough would determine which gauge groups each and every matter field is charged under and therefore uncover the full structure of the model.

\section{References}

1. ATLAS Collaboration, G. Aad et al., Observation of a new particle in the search for the Standard Model Higgs boson with the ATLAS detector at the LHC. Phys. Lett. B 716, 1-29 (2012). [arXiv: 1207.7214]

2. CMS Collaboration, S. Chatrchyan et al., Observation of a new boson at a mass of $125 \mathrm{GeV}$ with the CMS experiment at the LHC. Phys. Lett. B 716, 30-61 (2012). [arXiv:1207.7235]

3. P. Batra, A. Delgado, D.E. Kaplan, T.M. Tait, The Higgs mass bound in gauge extensions of the minimal supersymmetric standard model. JHEP 0402, 043 (2004). [hep-ph/0309149]

4. M. Dine, N. Seiberg, S. Thomas, Higgs physics as a window beyond the MSSM (BMSSM). Phys. Rev. D 76, 095004 (2007). [arXiv:0707.0005]

5. K. Blum, R.T. D'Agnolo, J. Fan, Natural SUSY predicts: Higgs couplings. JHEP 1301, 057 (2013). [arXiv:1206.5303]

6. C. Csaki, J. Erlich, C. Grojean, G.D. Kribs, 4-D constructions of supersymmetric extra dimensions and gaugino mediation. Phys. Rev. D 65, 015003 (2002). [hep-ph/0106044]

7. H. Cheng, D. Kaplan, M. Schmaltz, W. Skiba, Deconstructing gaugino mediation. Phys. Lett. B 515, 395-399 (2001). [hep-ph/0106098]

8. P. Batra, A. Delgado, D.E. Kaplan, T.M. Tait, Running into new territory in SUSY parameter space. JHEP 0406, 032 (2004), [hep-ph/0404251]

9. A. Delgado, Raising the Higgs mass in supersymmetric models. hep-ph/0409073

10. A. De Simone, J. Fan, M. Schmaltz, W. Skiba, Low-scale gaugino mediation, lots of leptons at the LHC. Phys. Rev. D 78, 095010 (2008). [arXiv:0808.2052]

11. A.D. Medina, N.R. Shah, C.E. Wagner, A Heavy Higgs and a Light Sneutrino NLSP in the MSSM with Enhanced SU(2) D-terms. Phys. Rev. D 80, 015001 (2009). [arXiv:0904.1625]

12. M. McGarrie, General gauge mediation and deconstruction. JHEP 1011, 152 (2010). [arXiv: 1009.0012]

13. N. Craig, D. Green, A. Katz, (De)Constructing a natural and flavorful supersymmetric standard model. JHEP 1107, 045 (2011). [arXiv:1103.3708]

14. R. Auzzi, A. Giveon, S.B. Gudnason, T. Shacham, A light stop with flavor in natural SUSY. JHEP 1301, 169 (2013). [arXiv:1208.6263]

15. R. Huo, G. Lee, A.M. Thalapillil, C.E. Wagner, $S U(2) \otimes S U(2)$ gauge extensions of the MSSM revisited. Phys. Rev. D 87, 055011 (2013). [arXiv:1212.0560]

16. R.T. D'Agnolo, E. Kuflik, M. Zanetti, Fitting the Higgs to natural SUSY. JHEP 1303, 043 (2013). [arXiv:1212.1165]

17. F. Staub, SARAH. [arXiv:0806.0538]

18. F. Staub, Automatic calculation of supersymmetric renormalization group equations and self energies. Comput. Phys. Commun. 182, 808-833 (2011). [arXiv:1002.0840] 
19. F. Staub, SARAH 3.2: Dirac Gauginos, UFO output, and more. Comput. Phys. Commun. 184, 1792-1809 (2013). [arXiv:1207.0906]

20. D.J. Muller, S. Nandi, Top flavor: a separate SU(2) for the third family. Phys. Lett. B 383, 345-350 (1996). [hep-ph/9602390]

21. E. Malkawi, T.M. Tait, C. Yuan, A model of strong flavor dynamics for the top quark. Phys. Lett. B 385, 304-310 (1996). [hep-ph/9603349]

22. E. Malkawi, C. Yuan, New physics in the third family and its effect on low-energy data. Phys. Rev. D 61, 015007 (2000). [hep-ph/9906215]

23. R. Auzzi, A. Giveon, The sparticle spectrum in minimal gauginogauge mediation. JHEP 1010, 088 (2010). [arXiv:1009.1714]

24. M. McGarrie, Hybrid gauge mediation. JHEP 1109, 138 (2011). [arXiv:1101.5158]

25. N. Craig, S. Dimopoulos, T. Gherghetta, Split families unified. JHEP 1204, 116 (2012). [arXiv:1203.0572]

26. B. Allanach, SOFTSUSY: a program for calculating supersymmetric spectra. Comput. Phys. Commun. 143, 305-331 (2002). [hep-ph/0104145]

27. A. Bharucha, A. Goudelis, M. McGarrie, Electroweak quiver gauge model (2013) (see supplementary material)

28. H. Baer, F.E. Paige, S.D. Protopopescu, X. Tata, Simulating supersymmetry with ISAJET 7.0/ISASUSY 1.0. [hep-ph/9305342]

29. A. Djouadi, J.-L. Kneur, G. Moultaka, SuSpect: a Fortran code for the supersymmetric and Higgs particle spectrum in the MSSM. Comput. Phys. Commun. 176, 426-455 (2007). [hep-ph/0211331]

30. W. Porod, SPheno, a program for calculating supersymmetric spectra, SUSY particle decays and SUSY particle production at e+ e- colliders. Comput. Phys. Commun. 153, 275-315 (2003). [hep-ph/0301101]]

31. P.Z. Skands, B. Allanach, H. Baer, C. Balazs, G. Belanger et al., SUSY Les Houches accord: interfacing SUSY spectrum calculators, decay packages, and event generators. JHEP 0407, 036 (2004). [hep-ph/0311123]

32. B. Allanach, C. Balazs, G. Belanger, M. Bernhardt, F. Boudjema, et al., SUSY Les Houches Accord 2. Comput. Phys. Commun. 180, 8-25 (2009). [arXiv:0801.0045]

33. W. Porod, F. Staub, SPheno 3.1: Extensions including flavour, CPphases and models beyond the MSSM. Comput. Phys. Commun. 183, 2458-2469 (2012). [arXiv:1104.1573]

34. A. Maloney, A. Pierce, J.G. Wacker, D-terms, unification, and the Higgs mass. JHEP 0606, 034 (2006). [hep-ph/0409127]

35. M. Drees, R. Godbole, P. Roy, Theory and phenomenology of sparticles: an account of four-dimensional $\mathrm{N}=1$ supersymmetry in high energy physics

36. M. Bando, T. Kugo, K. Yamawaki, Nonlinear realization and hidden local symmetries. Phys. Rep. 164, 217-314 (1988)

37. N. Arkani-Hamed, A.G. Cohen, H. Georgi, (De)constructing dimensions. Phys. Rev. Lett. 86, 4757-4761 (2001). [hep-th/0104005]

38. C.T. Hill, S. Pokorski, J. Wang, Gauge invariant effective Lagrangian for Kaluza-Klein modes. Phys. Rev. D 64, 105005 (2001). [hep-th/0104035]

39. N. Arkani-Hamed, A.G. Cohen, H. Georgi, Electroweak symmetry breaking from dimensional deconstruction. Phys. Lett. B 513, 232240 (2001). [hep-ph/0105239]

40. M. McGarrie, General resonance mediation. JHEP 1303, 093 (2013). [arXiv:1207.4484]

41. M. McGarrie, 5D maximally supersymmetric Yang-Mills in 4D superspace: applications. JHEP 1304, 161 (2013). [arXiv:1303.4534]

42. M. McGarrie, D.C. Thompson, Warped general gauge mediation. Phys. Rev. D 82, 125034 (2010). [arXiv:1009.4696]

43. M. McGarrie, Holography for general gauge mediation. JHEP 1302, 132 (2013). [arXiv:1210.4935]
44. M. McGarrie, R. Russo, General gauge mediation in 5D. Phys. Rev. D 82, 035001 (2010). [arXiv:1004.3305]

45. T. Hahn, Generating Feynman diagrams and amplitudes with FeynArts 3. Comput. Phys. Commun. 140, 418-431 (2001). [hep-ph/0012260]

46. T. Hahn, C. Schappacher, The implementation of the minimal supersymmetric standard model in FeynArts and FormCalc. Comput. Phys. Commun. 143, 54-68 (2002). [hep-ph/0105349]

47. T. Hahn, M. Perez-Victoria, Automatized one loop calculations in four-dimensions and D-dimensions. Comput. Phys. Commun. 118, 153-165 (1999). [hep-ph/9807565]

48. A. Pukhov, E. Boos, M. Dubinin, V. Edneral, V. Ilyin, et al., CompHEP: a package for evaluation of Feynman diagrams and integration over multiparticle phase space. [hep-ph/9908288]

49. P. Bechtle, O. Brein, S. Heinemeyer, G. Weiglein, K.E. Williams, HiggsBounds: confronting arbitrary Higgs sectors with exclusion bounds from LEP and the Tevatron. Comput. Phys. Commun. 181, 138-167 (2010). [arXiv:0811.4169]

50. P. Bechtle, O. Brein, S. Heinemeyer, G. Weiglein, K.E. Williams, HiggsBounds 2.0.0: confronting neutral and charged Higgs sector predictions with exclusion bounds from LEP and the Tevatron. Comput. Phys. Commun. 182, 2605-2631 (2011). [arXiv:1102.1898]

51. P. Bechtle, O. Brein, S. Heinemeyer, O. Stal, T. Stefaniak, et al., Recent developments in Higgs bounds and a preview of Higgs signals. PoS CHARGED2012 024 (2012). [arXiv:1301.2345]

52. W. Kilian, T. Ohl, J. Reuter, WHIZARD: simulating multi-particle processes at LHC and ILC. Eur. Phys. J. C 71, 1742 (2011). [arXiv:0708.4233]

53. G. Belanger, F. Boudjema, A. Pukhov, A. Semenov, MicrOMEGAs 2.0: a program to calculate the relic density of dark matter in a generic model. Comput. Phys. Commun. 176, 367-382 (2007). [hep-ph/0607059]

54. J. Camargo-Molina, B. O'Leary, W. Porod, F. Staub, Vevacious: a tool for finding the global minima of one-loop effective potentials with many scalars. [arXiv:1307.1477]

55. G. Degrassi, P. Slavich, F. Zwirner, On the neutral Higgs boson masses in the MSSM for arbitrary stop mixing. Nucl. Phys. B 611, 403-422 (2001). [hep-ph/0105096]

56. A. Brignole, G. Degrassi, P. Slavich, F. Zwirner, On the $\mathrm{O}(\operatorname{alpha}(\mathrm{t}) * * 2)$ two loop corrections to the neutral Higgs boson masses in the MSSM. Nucl. Phys. B 631, 195-218 (2002). [hep-ph/0112177]

57. A. Brignole, G. Degrassi, P. Slavich, F. Zwirner, On the two loop sbottom corrections to the neutral Higgs boson masses in the MSSM. Nucl. Phys. B 643, 79-92 (2002). [hep-ph/0206101]

58. A. Dedes, P. Slavich, Two loop corrections to radiative electroweak symmetry breaking in the MSSM. Nucl. Phys. B 657, 333-354 (2003). [hep-ph/0212132]

59. N. Craig, A. Katz, A supersymmetric Higgs sector with chiral Dterms. JHEP 1305, 015 (2013). [arXiv: 1212.2635]

60. ATLAS Collaboration, Physics at a high-luminosity LHC with ATLAS. [arXiv:1307.7292]

61. CMS Collaboration, Projected performance of an upgraded CMS detector at the LHC and HL-LHC: contribution to the snowmass process. [arXiv:1307.7135]

62. CMS Collaboration, S. Chatrchyan et al., Observation of a new boson with mass near $125 \mathrm{GeV}$ in pp collisions at $\operatorname{sqrt}(\mathrm{s})=7$ and 8 TeV. JHEP 06, 081 (2013). [arXiv:1303.4571]

63. G. Degrassi, S. Heinemeyer, W. Hollik, P. Slavich, G. Weiglein, Towards high precision predictions for the MSSM Higgs sector. Eur. Phys. J. C 28, 133-143 (2003). [hep-ph/0212020]

64. A. Arbey, M. Battaglia, A. Djouadi, F. Mahmoudi, The Higgs sector of the phenomenological MSSM in the light of the Higgs boson discovery. JHEP 1209, 107 (2012). [arXiv:1207.1348] 
65. Particle Data Group Collaboration, J. Beringer et al., Review of particle physics (RPP), Phys. Rev. D 86, 010001 (2012)

66. ATLAS Collaboration, Search for squarks and gluinos with the atlas detector in final states with jets and missing transverse momentum and $20.3 \mathrm{fb}^{-1}$ of $\sqrt{s}=8$ tev proton-proton collision data, Tech. Rep. ATLAS-CONF-2013-047, CERN, Geneva (2013)

67. CMS Collaboration, S. Chatrchyan et al., Search for new physics in the multijet and missing transverse momentum final state in proton-proton collisions at $\sqrt{s}=7 \mathrm{TeV}$. Phys. Rev. Lett. 109, 171803 (2012). [arXiv:1207.1898]

68. H. Baer, V. Barger, D. Mickelson, How conventional measures overestimate electroweak fine-tuning in supersymmetric theory. [arXiv:1309.2984]

69. ALEPH, DELPHI, L3 and OPAL Collaboration, LEPSUSYWG, notes LEPSUSYWG/02-04.1 and LEPSUSYWG/01-03.1 (2001)

70. ALEPH Collaboration, R. Barate et al., Search for gauge mediated SUSY breaking topologies at $S^{(1 / 2)}$ similar to $189-G e V$. Eur. Phys. J. C 16, 71-85 (2000)

71. ALEPH Collaboration, R. Barate et al., Search for supersymmetric particles in $e^{+} e^{-}$collisions at $\sqrt{s}$ up to $202-\mathrm{GeV}$ and mass limit for the lightest neutralino. Phys. Lett. B 499, 67-84 (2001). [hep-ex/0011047]

72. ALEPH Collaboration, D. Decamp et al., Searches for new particles in $Z$ decays using the ALEPH detector. Phys. Rep. 216, 253-340 (1992)

73. P. Bechtle, S. Heinemeyer, O. Stål, T. Stefaniak, G. Weiglein, Higgs signals: confronting arbitrary Higgs sectors with measurements at the Tevatron and the LHC. [arXiv:1305.1933]

74. Heavy Flavor Averaging Group Collaboration, Y. Amhis et al., Averages of B-Hadron, C-Hadron, and tau-lepton properties as of early 2012. [arXiv:1207.1158]

75. LHCb Collaboration, R. Aaij et al., First evidence for the decay $B_{s}^{0} \rightarrow \mu^{+} \mu^{-}$. Phys. Rev. Lett. 110, 021801 (2013). [arXiv: 1211.2674]

76. H.E. Haber, R. Hempfling, A.H. Hoang, Approximating the radiatively corrected Higgs mass in the minimal supersymmetric model. Z. Phys. C 75, 539-554 (1997). [hep-ph/9609331]

77. R. Benbrik, M. Gomez, Bock, S. Heinemeyer, O. Stal, G. Weiglein, et al., Confronting the MSSM and the NMSSM with the discovery of a signal in the two photon channel at the LHC. Eur. Phys. J. C 72, 2171 (2012). [arXiv:1207.1096]

78. P. Bechtle, S. Heinemeyer, O. Stal, T. Stefaniak, G. Weiglein et al., MSSM interpretations of the LHC discovery: light or heavy Higgs? Eur. Phys. J. C 73, 2354 (2013). [arXiv:1211.1955]

79. ATLAS Collaboration, G. Aad et al., Measurements of Higgs boson production and couplings in diboson final states with the ATLAS detector at the LHC. Phys. Lett. B (2013). [arXiv:1307.1427]

80. CMS Collaboration, Combination of standard model Higgs boson searches and measurements of the properties of the new boson with a mass near $125 \mathrm{GeV}$, Tech. Rep. CMS-PAS-HIG-12-045, CERN, Geneva (2012)
81. H. Baer, T. Barklow, K. Fujii, Y. Gao, A. Hoang, et al., The International Linear Collider Technical Design Report, Vol. 2, Physics. [arXiv:1306.6352]

82. ATLAS Collaboration, Coupling properties of the new Higgslike boson observed with the ATLAS detector at the LHC, Tech. Rep. ATLAS-CONF-2012-127, ATLAS-COM-CONF-2012-161, CERN, Geneva (2012)

83. ATLAS Collaboration, G. Aad et al., Search for diphoton events with large missing transverse momentum in $7 \mathrm{TeV}$ proton-proton collision data with the ATLAS detector. Phys. Lett. B 718, 411-430 (2012). [arXiv:1209.0753]

84. ATLAS Collaboration, G. Aad et al., Search for supersymmetry in events with photons, bottom quarks, and missing transverse momentum in proton-proton collisions at a centre-of-mass energy of $7 \mathrm{TeV}$ with the ATLAS detector. Phys. Lett. B 719, 261-279 (2013). [arXiv:1211.1167]

85. G. Giudice, R. Rattazzi, Theories with gauge mediated supersymmetry breaking. Phys. Rep. 322, 419-499 (1999). [hep-ph/9801271]

86. ATLAS Collaboration, G. Aad et al., Search for non-pointing photons in the diphoton and $E_{T}^{m}$ iss final state in $\operatorname{sqrt}(\mathrm{s})=7 \mathrm{TeV}$ proton-proton collisions using the ATLAS detector. Phys. Rev. D 88, 012001 (2013). [arXiv:1304.6310]

87. CMS Collaboration, S. Chatrchyan et al., Search for long-lived particles decaying to photons and missing energy in proton-proton collisions at $\sqrt{s}=7 \mathrm{TeV}$, Phys. Lett. B 722, 273-294 (2013). [arXiv: 1212.1838]

88. CMS Collaboration, S. Chatrchyan et al., Search for new physics in events with photons, jets, and missing transverse energy in $p p$ collisions at $\sqrt{s}=7$ TeV. JHEP 1303, 111 (2013). [arXiv:1211.4784]

89. B. Allanach, M. Battaglia, G. Blair, M.S. Carena, A. De Roeck et al., The snowmass points and slopes: benchmarks for SUSY searches. Eur. Phys. J. C 25, 113-123 (2002). [hep-ph/0202233]

90. ATLAS Collaboration, Search for supersymmetry in final states with jets, missing transverse momentum and a $Z$ boson at $\sqrt{s}=8$ $\mathrm{TeV}$ with the ATLAS detector, Tech. Rep. ATLAS-CONF-2012152, CERN, Geneva (2012)

91. CMS Collaboration, A search for anomalous production of events with three or more leptons using 19.5/fb of $\operatorname{sqrt}(\mathrm{s})=8 \mathrm{TeV}$ LHC data, Tech. Rep. CMS-PAS-SUS-13-002, CERN, Geneva (2013)

92. ATLAS Collaboration, Search for supersymmetry in events with large missing transverse momentum, jets, and at least one tau lepton in $21 \mathrm{fb}^{-1}$ of $\sqrt{s}=8 \mathrm{TeV}$ proton-proton collision data with the ATLAS detector, Tech. Rep. ATLAS-CONF-2013-026, CERN, Geneva (2013)

93. A. Bharucha, S. Heinemeyer, F. von der Pahlen, Direct charginoneutralino production at the LHC: interpreting the exclusion limits in the complex MSSM. [arXiv:1307.4237]

94. R. Auzzi, A. Giveon, S.B. Gudnason, Mediation of supersymmetry breaking in quivers. JHEP 1112, 016 (2011). [arXiv:1110.1453] 